\title{
EXTENSION OF LIPSCHITZ MAPS INTO 3-MANIFOLDS*
}

\author{
SERGEI BUYALO ${ }^{\dagger}$ AND VIKTOR SCHROEDER ${ }^{\ddagger}$
}

\begin{abstract}
We prove that the universal covering $Y$ of a closed nonpositively curved 3-dimensional Riemannian manifold possesses the following Lipschitz extension property: there exists a constant $c \geq 1$ such that every $\lambda$-Lipschitz map $f: S \rightarrow Y$ defined on a subset $S$ of an arbitrary metric space $X$ has a $c \lambda$-Lipschitz extension $\bar{f}: X \rightarrow Y$.
\end{abstract}

1. Introduction. We say that a metric space $Y$ has the Lipschitz extension property $(L)$ if there exists a constant $c \geq 1$ such that every $\lambda$-Lipschitz map $f$ : $S \rightarrow Y$ defined on an arbitrary subset $S$ of some metric space $X$ can be extended to a $c \lambda$-Lipschitz map $\bar{f}: X \rightarrow Y$.

Obviously, to have property (L) is a bilipschitz invariant of $Y$. One can prove that the Lipschitz extension property implies that $Y$ is contactible. A space with property (L) also satisfies a quadratic isoperimetric inequality for closed curves, i.e. a closed curve of length $l$ in $Y$ can be spanned by a surface with area $\leq c^{\prime} l^{2}$. This follows from the fact that the arclength parametrization of the curve defined on the circle $S_{r}^{1} \subset \mathbb{R}^{2}$ of radius $r=\frac{l}{2 \pi}$ is Lipschitz and can be extended to a Lipschitz map defined on the disc.

A classical result of McShane [M] states that $\mathbb{R}$ has the property (L) with constant $c(\mathbb{R})=1$. Applying this result to the coordinate functions, $\mathbb{R}^{n}$ has property $(\mathrm{L})$ with constant $c\left(\mathbb{R}^{n}\right)=\sqrt{n}$. Lang [L] showed that the optimal constant for $\mathbb{R}^{n}$ has to depend on $n$ and that (L) is not valid for an infinite-dimensional Hilbert space.

In [LPS] it is proved that the following three classes of Hadamard spaces have the property (L)

(1) the 2-dimensional Hadamard manifolds;

(2) the class of Gromov-hyperbolic Hadamard manifolds whose curvature is bounded by $-b^{2} \leq K \leq 0$

(3) the class of homogeneous Hadamard manifolds and euclidean Tits buildings.

In this paper we study the validity of (L) for 3-dimensional spaces. Let us first investigate the standard homogeneous 3-dimensional geometries:

$S^{3}, \mathbb{R}^{3}, \mathrm{H}^{3}, S^{2} \times \mathbb{R}, \mathrm{H}^{2} \times \mathbb{R}, N I L, S O L, \widetilde{P S L}_{2}(\mathbb{R})$.

Since $S^{3}$ and $S^{2} \times \mathbb{R}$ are homotopically nontrivial, they do not satisfy the extension property, while $\mathbb{R}^{3}, \mathrm{H}^{3}$ and $\mathrm{H}^{2} \times \mathbb{R}$ satisfies (L) by McShane's result and the case (3) above. Since $\widetilde{P S L}(\mathbb{R})$ is bilipschitz to $\mathrm{H}^{2} \times \mathbb{R}$ (this observation is due to Epstein, Mess and Gersten according to [KLe]) the property (L) is satisfied. On the other hand NIL and SOL do not share (L) since they do not allow quadratic isoperimetric inequalities (see [Eetal]).

We show that the property (L) holds for a large class of simply connected 3manifolds. Our results can be summarized by the following two theorems.

TheOREM A. Let $Y$ be the universal covering of a nonpositively curved, closed Riemannian 3-manifold. Then $Y$ satisfies (L).

*Received January 31, 2001; accepted for publication February 8, 2001.

†Steklov Institute of Mathematics, Fontanka 27, 191011, St. Petersburg, Russia (buyalo@pdmi.ras.ru). This author was partially supported by RFFI Grant 99-01-00104 and SNF Grant 20-57151.99

$\ddagger$ Institut für Mathematik, Universität Zürich, Winterthurer Strasse 190, CH-8057 Zürich, Switzerland (vschroed@math.unizh.ch). 
Theorem B. Let $M$ be a metric space homeomorphic to a Haken manifold (possibly with boundary) with zero Euler characteristic which is not of type NIL or SOL. Then the universal covering $Y$ of $M$ satisfies (L).

We recall that a Haken manifold is a compact irreducible 3-dimensional manifold which contains a closed embedded 2-sided surface whose fundamental group is infinite and injects via the canonical inclusion homomorphism. Besides quotients of NIL and $S O L$ Theorem B includes other classes of manifolds which cannot carry metrics of nonpositive curvature. By [BK], [Le] there are graphmanifolds which cannot carry metrics of nonpositive sectional curvature.

Actually Theorem A can be reduced to Theorem B in the following way: Let $Y$ be the universal covering of a closed nonpositively curved 3-manifold $M$. We represent $M$ as $Y / \Gamma$ where $\Gamma$ is the group of decktransformations on $Y$. By a result of Eberlein [Eb], $Y$ is either Gromov-hyperbolic or contains a two-dimensional totally geodesic flat plane $F \subset Y$. If $Y$ is hyperbolic, then $Y$ is in the class (2) considered above and the property (L) follows. If $Y$ contains a flat plane, then by [B], [S] there exists also a closed flat plane. Hence there exists a flat plane $F \subset Y$ such that the group $\Gamma_{F}=\{\gamma \in \Gamma: \gamma(F)=F\}$ operates with compact quotient on $F$. The proof of this result also shows that there exists indeed a flat $F \subset Y$ which is embedded into $M$, i.e. for all $\gamma \in \Gamma$ either $\gamma(F)=F$ or $\gamma(F) \cap F=\emptyset$. Then the set of flat planes $\{\gamma(F): \gamma \in \Gamma\}$ divide $Y$ into convex subsets (blocks) and this decomposition is invariant under $\Gamma$. One can colour the blocks with two colours such that adjacent blocks have different colours. Clearly a finite index subgroup of $\Gamma$ leaves the colouring invariant and hence $M$ is finitely covered by a nonpositively curved manifold which contains an embedded 2-sided torus whose fundamental group injects. Thus $M$ is finitely covered by a Haken manifold (which is not of type NIL or SOL).

In order to prove Theorem $B$ we use results of [Le] and [KLe] to show that the manifold $Y$ is bilipschitz to a convex subset of a 3-dimensional Hadamard space which is built out of very special blocks corresponding to the geometric decomposition of $M$. Thus we have to prove property (L) only for this special class of Hadamard spaces, which we call Hadamard spaces with a $S H$-block structure. For details of this structure and the existence of the bilipschitz map see section 4 .

To prove (L) for a Hadamard space $Y$ with $S H$-block structure, we use the approach from [LPS] which applies for all Hadamard spaces $Y$ :

Let $f: S \rightarrow Y$ be a $\lambda$-Lipschitz map. Then one can associate to every $x \in X$ a bounded closed convex subset $A(x):=\bigcap_{s \in S} B(f(s), 2 \lambda \operatorname{dist}(x, s)) \subset Y$. Note that $A(x)=\{f(x)\}$ for $x \in S$. In [LPS] it is shown that the map $A: X \rightarrow \mathcal{C}$, where $\mathcal{C}$ is the space of bounded closed convex subsets of $Y$ endowed with the Hausdorff metric Hd, is Lipschitz with constant $2 \sqrt{2} \lambda$. In order to obtain the required extension $\bar{f}: X \rightarrow Y$ one has to compose $A$ with a Lipschitz-retraction $R: \mathcal{C} \rightarrow Y$, where we identify $Y$ canonically with a subset of $\mathcal{C}$. Note that the existence of the Lipschitz retraction $R$ is a special case of the general problem.

In general the existence of $R$ is not clear for arbitrary Hadamard spaces, we will show however:

Theorem C. Let $Y$ be a Hadamard space with a $S H$-block structure and let $\mathcal{C}$ be the set of bounded closed convex subsets of $Y$ endowed with the Hausdorff distance. Identify $Y \subset \mathcal{C}$ via the canonical inclusion $y \mapsto\{y\}$. Then there exists a Lipschitz retraction $R: \mathcal{C} \rightarrow Y$.

Indeed we will prove only a weaker statement, namely the existence of a quasiLipschitz retraction: 
THEOREM D. Let $Y$ be a Hadamard space with a $S H$-block structure. Then there are constants $L$ and $l$ and a map $R: \mathcal{C} \rightarrow Y$ such that dist $\left(R(A), R\left(A^{\prime}\right)\right) \leq$ $L \operatorname{Hd}\left(A, A^{\prime}\right)+l$ for every $A, A^{\prime} \in \mathcal{C}$ and dist $(y, R(y)) \leq l$ for each $y \in Y$.

Using a main result of [LPS] we can deduce Theorem $C$ from the weaker Theorem D: first restrict the $(L, l)$-Lipschitz map $R$ to a set $N$, which is a maximal discrete subset in $\mathcal{C} \backslash Y$ with the property that $\operatorname{Hd}\left(A, A^{\prime}\right) \geq l$ whenever $A \in N, A^{\prime} \in Y \cup N$. Then we define $R^{\prime}: N \cup Y \rightarrow Y$ by $R^{\prime}|N=R| N, R^{\prime} \mid Y=\operatorname{id}_{Y}$. The map $R^{\prime}$ is $L^{\prime}$-Lipschitz and by the local extension result [LPS], Theorem 5.3, this map can be extended to a Lipschitz map on $\mathcal{C}$. Note that $Y$ is geodesically complete and satisfies the local doubling property required in the cited Theorem 5.3, since $Y$ is easily seen to be bilipschitz to a Hadamard manifold with bounded sectional curvature.

The aim of the rest of the paper is the proof of Theorem D. In section 3 we discuss the geometry of the space $\mathcal{C}$ of bounded closed convex subsets of an arbitrary locally compact Hadamard space in more detail. In section 4 we discuss Hadamard spaces with a block structure and more particular Hadamard spaces with a $S H$-block structure. In section 5 we construct the $(L, l)$-Lipschitz retraction of Theorem D.

We would like to thank Thomas Schick for the remark that property (L) implies the contractibility of the space.

2. Preliminaries. We recall some general facts from the theory of Hadamard spaces, see $[\mathrm{BrH}]$. Let $(Y$, dist) be a Hadamard space, i.e. a complete geodesic metric space satisfying $\operatorname{CAT}(0)$ inequality which means that triangles are thinner than in euclidian space. A Hadamard space is called CAT(-1), if triangles are even thinner than comparison triangles in hyperbolic space. The unique geodesic arc between two points $y$ and $y^{\prime}$ is denoted by $y y^{\prime}$. For every bounded nonempty subset $A \subset Y$ there is a uniquely determined smallest closed ball containing $A$. Its center is called the circumcenter of $A$. With $\operatorname{diam} A$ we denote the diameter of $A$. If $A, A^{\prime}$ are closed bounded subsets of $Y$ let

$$
\operatorname{Hd}\left(A, A^{\prime}\right):=\inf \left\{\varepsilon>0: A \subset U_{\varepsilon}\left(A^{\prime}\right), A^{\prime} \subset U_{\varepsilon}(A)\right\}
$$

be the Hausdorff distance, where $U_{\varepsilon}(A):=\{y \in Y: \operatorname{dist}(y, A) \leq \varepsilon\}$. A subset $A \subset Y$ is convex, if $A$ contains $y y^{\prime}$ for all points $y, y^{\prime} \in A$. For a closed convex subset $A \subset Y$ the distance function $\operatorname{dist}(\cdot, A)$ is convex and for every $y \in Y$ there is a unique point $p_{A}(y) \in A$ closest to $y . p_{A}: Y \rightarrow A$ is called the metric projection onto $A$.

\section{The Space of Convex Subsets in a Hadamard Space.}

3.1. Convex hull and convex projection. Let $Y$ be a Hadamard space, $\mathcal{B}$ the space of closed, bounded subsets in $Y$ equipped with the Hausdorff metric denoted by $\mathrm{Hd}, \mathcal{C} \subset \mathcal{B}$ consists of the convex subsets. There is the canonical projection conv : $\mathcal{B} \rightarrow \mathcal{C}$ which associates to each $B \in \mathcal{B}$ its closed convex hull $\operatorname{conv}(B)$.

Lemma 3.1. The map conv : $\mathcal{B} \rightarrow \mathcal{C}$ is 1-Lipschitz and does not change the diameter.

Proof. Connecting $b, b^{\prime} \in B \in \mathcal{B}$ by the geodesic segment increases neither diam $B$ nor the Hausdorff distance to any $B^{\prime} \in \mathcal{B}$ by convexity of the distance function. The claim follows since $\operatorname{conv}(B)$ coincides with closure of $\cup_{n} B_{n}$, where $B_{0}=B$ and $B_{n+1}$ is obtained from $B_{n}$ by connecting each pair of points $b, b^{\prime} \in B_{n}$ by the geodesic segment.

Given a closed, convex $C \subset Y$, we have the metric projection $p_{C}: Y \rightarrow C$ which is a 1-Lipschitz map. The map $\bar{p}_{C}: \mathcal{C} \rightarrow \mathcal{C}, \bar{p}_{C}(A)=$ conv op $p_{C}(A)$ is called the convex projection on $C$. 
Lemma 3.2. Let $C$ be a closed, convex subset in $Y$. Then the convex projection $\bar{p}_{C}: \mathcal{C} \rightarrow \mathcal{C}$ is 1-Lipschitz.

Proof. Since $p_{C}$ is 1-Lipschitz, the induced map $\widehat{p}_{C}: \mathcal{B} \rightarrow \mathcal{B}$ is 1-Lipschitz too. Hence, $\bar{p}_{C}$ is 1-Lipschitz by Lemma 3.1 .

Lemma 3.3. The diameter diam $: \mathcal{C} \rightarrow \mathbb{R}$ is a 2-Lipschitz function.

Proof. Given $A, A^{\prime} \in \mathcal{C}$, we take $a_{0}, a_{1} \in A$ which approximate $\operatorname{diam} A$ up to an arbirarily small error (we do not suppose that $A$ is compact). Let $a_{0}^{\prime}, a_{1}^{\prime} \in A^{\prime}$ be closest points to $a_{0}, a_{1}$ respectively. Then

$$
\operatorname{diam} A^{\prime} \geq \operatorname{dist}\left(a_{0}^{\prime}, a_{1}^{\prime}\right) \geq \operatorname{dist}\left(a_{0}, a_{1}\right)-2 \operatorname{Hd}\left(A, A^{\prime}\right) .
$$

Hence, the claim.

3.2. Geodesics in $\mathcal{C}$. Here we study in more detail the space $\mathcal{C}=\mathcal{C}(Y)$ assuming that the Hadamard space $Y$ is locally compact. The points of $Y$ are elements of $\mathcal{C}$, and this gives the canonical isometric embedding $Y \subset \mathcal{C}$. We identify $Y$ with its image in $\mathcal{C}$.

The space $\mathcal{C}$ is a geodesic space (see Proposition 3.5), and one can show that the Hausdorff metric is convex in some weak sense. Given $y, y^{\prime} \in Y$, there is a unique midpoint $z \in \mathcal{C}$ between $y, y^{\prime}$, which coincides with the midpoint of the segment $y y^{\prime} \subset Y$. This follows from the fact that the closed balls in $Y$ of radius $\operatorname{dist}\left(y, y^{\prime}\right) / 2$, centered at $y, y^{\prime}$ respectively, have a unique point in common, namely, $z$. This argument shows that $Y$ is a (closed) convex subset in $\mathcal{C}$. Moreover, the canonical map circ $: \mathcal{C} \rightarrow Y$ given by the circumcenter of a convex set has the property

$$
\operatorname{Hd}(A, \operatorname{circ}(A))=\inf _{y \in Y} \operatorname{Hd}(A, y)
$$

i.e., circ is a metric projection. However, already for $Y=\mathbb{R}^{2}$ examples show (see [LPS]) that this map is not Lipschitz.

The following lemma is a version of Theorem 1.8.2 from [Sch] where only the case $Y=\mathbb{R}^{n}$ is considered.

LEMma 3.4. The space $\mathcal{C}$ is complete.

Proof. Let $\left\{A_{i}\right\} \subset \mathcal{C}$ be a Cauchy sequence. Then $B_{j}=\overline{\cup_{i \geq j} A_{i}} \in \mathcal{B}$ for each $j \geq 1$. Furthermore, $\left\{B_{j}\right\}$ decreases, $B_{j+1} \subset B_{j}$. Since $Y$ is locally compact and hence proper, the set $B=\cap_{j} B_{j}$ is not empty. We show that $\operatorname{Hd}\left(A_{i}, B\right) \leq \varepsilon$ for each $\varepsilon>0$ and all sufficiently large $i$. First, we note that $\operatorname{Hd}\left(A_{i}, A_{j}\right) \leq \varepsilon$ for all sufficiently large $i, j$. It follows that $B_{j} \subset U_{\varepsilon}\left(A_{i}\right)$ and thus $B \subset U_{\varepsilon}\left(A_{i}\right)$. Similarly, $A_{i} \subset U_{\varepsilon}\left(B_{j}\right)$, and we obtain $A_{i} \subset U_{\varepsilon}(B)$. Then $\operatorname{Hd}\left(A_{i}, \widehat{B}\right) \leq \varepsilon$ for $\widehat{B}=\operatorname{conv}(B)$ by Lemma 3.1. Thus $\widehat{B}=\lim _{i} A_{i}$.

Proposition 3.5. Let $Y$ be a locally compact Hadamard space. Then $\mathcal{C}=\mathcal{C}(Y)$ is a geodesic space.

Proof. We first prove the existence of a midpoint between any two $A, A^{\prime} \in \mathcal{C}$. This is true for any Hadamard space $Y$ without the requirement to be locally compact.

Let $B \subset Y$ be the set of the midpoints of all geodesic segments $a a^{\prime} \subset Y$ with $a \in A, a^{\prime} \in A^{\prime}$. We put $\lambda=\frac{1}{2} \operatorname{Hd}\left(A, A^{\prime}\right)$ and assume that there exists $b \in B$ with $\operatorname{dist}(b, A)>\lambda$. This $b$ is the midpoint of a segment $a a^{\prime}$ with $a \in A, a^{\prime} \in A^{\prime}$. Since $A$ is convex, the distance function to $A$ is convex. Thus $\operatorname{dist}\left(a^{\prime}, A\right) \geq 2 \operatorname{dist}(b, A)$ because $\operatorname{dist}(a, A)=0$. Hence, $\operatorname{dist}\left(a^{\prime}, A\right)>\operatorname{Hd}\left(A, A^{\prime}\right)$ contradicting the definition of $\operatorname{Hd}\left(A, A^{\prime}\right)$. This shows that $B$ lies in the closed $\lambda$-neighbourhood of $A, U_{\lambda}(A)$. 
On the other hand, for each $a \in A$ there is $b \in B$ with $\operatorname{dist}(b, a) \leq \lambda$ : let $b$ be the midpoint of $a a^{\prime}$. where $a^{\prime} \in A^{\prime}$ is the closest point to $a$, thus $\operatorname{dist}\left(a, a^{\prime}\right) \leq 2 \lambda$. This shows that $A \subset U_{\lambda}(B)$. Thus $\operatorname{Hd}(B, A) \leq \lambda$ and, similarly, $\operatorname{Hd}\left(B, A^{\prime}\right) \leq \lambda$. By the triangle inequality $2 \lambda \leq \operatorname{Hd}(A, B)+\operatorname{Hd}\left(B, A^{\prime}\right) \leq 2 \lambda$ and hence

$$
\operatorname{Hd}(B, A)=\lambda=\operatorname{Hd}\left(B, A^{\prime}\right) .
$$

For the convex hull $\widehat{B}=\operatorname{conv}(B)$ we have $\operatorname{Hd}(\widehat{B}, A), \operatorname{Hd}\left(\widehat{B}, A^{\prime}\right) \leq \lambda$ by Lemma 3.1. Hence, $\operatorname{Hd}(\widehat{B}, A)=\lambda=\operatorname{Hd}\left(\widehat{B}, A^{\prime}\right)$ by the triangle inequality. Thus $\widehat{B}$ is a midpoint between $A$ and $A^{\prime}$.

By Lemma $3.4, \mathcal{C}$ is complete. It follows that $\mathcal{C}$ is geodesic.

REMARK 3.6. Two sets $A, A^{\prime} \in \mathcal{C}$ possess a unique midpoint in $\mathcal{C}$ only in exceptional cases, see [Sch]. Yet, the procedure described above gives the canonical geodesic segment between any two points in $\mathcal{C}$.

3.3. Geodesics in $\mathcal{C}$ associated with a distance function. Let $h: Y \rightarrow$ $\mathbb{R}$ be the distance function to a closed, convex subset $C \subset Y$. Then $h$ is convex and 1-Lipschitz. Furthermore, the sets $C_{t}=\{y \in Y: h(y) \leq t\}$ are convex and $\operatorname{Hd}\left(C_{t}, C_{t^{\prime}}\right)=\left|t^{\prime}-t\right|$ for each $t, t^{\prime} \geq 0$.

Given $A \in \mathcal{C}$, we let $t_{A}:=\inf \left\{t \geq 0: A \subset C_{t}\right\}, \bar{p}_{t}:=\bar{p}_{C_{t}}: \mathcal{C} \rightarrow \mathcal{C}$ be convex projections.

Lemma 3.7. For each $A \in \mathcal{C}, 0 \leq t \leq t_{A}$ we have

$$
\operatorname{Hd}\left(A, \bar{p}_{t}(A)\right)=t_{A}-t
$$

Proof. If $t_{A}=0$ then the claim is obvious. Otherwise $A \cap \partial C_{t_{A}} \neq \emptyset$ by the definition of $t_{A}$ and $\bar{p}_{t}(A) \subset C_{t}$, we have $\operatorname{Hd}\left(A, \bar{p}_{t}(A)\right) \geq t_{A}-t$. On the other hand, $\operatorname{Hd}\left(A, p_{t}(A)\right) \leq t_{A}-t$ because $A \subset C_{t_{A}}$ and by properties of the metric projection $p_{t}: Y \rightarrow C_{t}$. Using Lemma 3.1, we obtain $\operatorname{Hd}\left(A, \bar{p}_{t}(A)\right) \leq t_{A}-t$. $\square$

Using the distance function $h$, we construct geodesic paths in $\mathcal{C}$ as follows.

Proposition 3.8. For each $A \in \mathcal{C}$ there exists a unique path $\sigma_{A}:[0, \infty) \rightarrow \mathcal{C}$ with the properties:

(1) $\sigma_{A}(t)=A$ for all $t \geq t_{A}$;

(2) $\sigma_{A}(t)$ is the minimal convex subset in $C_{t}$ containing $\bar{p}_{t} \circ \sigma_{A}\left(t^{\prime}\right)$ for all $t^{\prime}>t$. Furthermore, the restriction $\sigma_{A} \mid\left[0, t_{A}\right]$ is a geodesic in $\mathcal{C}$.

Proof. We first show that $\sigma_{A}$ exists. By (1), it is already defined for all $t \geq t_{A}$. For dyadic numbers $D_{n}=\left\{k 2^{-n}: k=0,1, \ldots, 2^{n}\right\} \subset[0,1]$ we define by induction

$$
\gamma_{n}(1)=A, \quad \gamma_{n}\left(k 2^{-n}\right)=\bar{p}_{s t_{A}} \circ \gamma_{n}\left((k+1) 2^{-n}\right)
$$

where $s=k 2^{-n}, k=2^{n}-1, \ldots, 1,0$. Clearly, $\gamma_{n}(s) \in \mathcal{C}$ is the minimal convex subset in $C_{t}, t=s t_{A}$, containing $\bar{p}_{t} \circ \gamma_{n}\left(s^{\prime}\right)$ for all $s^{\prime}>s \in D_{n}$. It also follows from this definition and Lemma 3.7 that the map in $: D_{n} \rightarrow \mathcal{C}$ is a homothety with coefficient $t_{A}$. In particular, $\operatorname{Hd}\left(A, \gamma_{n}(s)\right) \leq t_{A}$ for each $s \in D_{n}$. On the other hand, $\bar{p}_{t}\left(A^{\prime}\right) \subset \bar{p}_{t} \circ \bar{p}_{t^{\prime}}\left(A^{\prime}\right)$ for each $A^{\prime} \in \mathcal{C}, t^{\prime} \geq t$. Thus $\gamma_{n+1}(s) \supset \gamma_{n}(s)$ for each $s \in D_{n}$. In other words, the sequence of convex sets, $\gamma_{n+p}(s), p \geq 1$ increases and all these sets lie in the $t_{A}$-neighbourhood of $A$. Thus there exists a limit

$$
\sigma_{A}\left(s t_{A}\right)=\lim _{p \rightarrow \infty} \gamma_{n+p}(s) \in \mathcal{C}
$$


for each $s \in D=\cup_{n} D_{n}$. This defines $\sigma_{A}$ on "dyadic" numbers in $\left[0, t_{A}\right]$. Clearly, $\sigma_{A}$ is isometric on this set and possesses property (2). Now, using Lemma 3.4, we extend $\sigma_{A}$ to an isometric map $\left[0, t_{A}\right] \rightarrow \mathcal{C}$, which possesses property (2).

Assume that there is another map $\sigma_{A}^{\prime}:[0, \infty) \rightarrow \mathcal{C}$ with properties (1), (2). Thus $\sigma_{A}^{\prime}$ coincides with $\sigma_{A}$ on $\left[t_{A}, \infty\right)$, in particular, $\sigma_{A}^{\prime}\left(t_{A}\right)=A$. It follows from the construction of $\sigma_{A}$ that $\sigma_{A}(t) \subset \sigma_{A}^{\prime}(t)$ for each $t \geq 0$ since $\sigma_{A}^{\prime}(t)$ contains $\bar{p}_{t} \circ \sigma_{A}^{\prime}\left(t^{\prime}\right)$ for all $t^{\prime}>t$ according (2). Then by minimality $\sigma_{A}^{\prime}=\sigma_{A}$.

Lemma 3.9. For every $A, A^{\prime} \in \mathcal{C}$, the function

$$
\varphi_{A, A^{\prime}}(t)=\operatorname{Hd}\left(\sigma_{A}(t), \sigma_{A^{\prime}}(t)\right)
$$

increases on $[0, \infty)$.

Proof. This immediately follows from Lemma 3.2 and the construction of $\sigma_{A}$.

\section{Hadamard Spaces with Block Structure.}

4.1. Block decomposition of $Y$ and the associated tree. We say that a 3-dimensional Hadamard space $Y$ has a block-structure, if it has a decomposition $Y=\cup_{v \in V} Y_{v}$ with the following properties: each block $Y_{v}$ is a closed, convex subset with non empty interior and geodesic boundary $\partial Y_{v}$, which is the countable union of disjoint 2-flats in $Y$. Every two blocks $Y_{v}, Y_{v^{\prime}}$ either are disjoint or have a common boundary component which separates them. We assume in addition that the minimal distance between different boundary components of $Y_{v}$ is at least 10 .

A boundary component of a block is called a wall. Each wall is adjacent to exactly two blocks and is a convex subset of $Y$ isometric to $\mathbb{R}^{2}$.

Let $T=T(Y)$ be the graph dual to the decomposition $Y=\cup_{v \in V} Y_{v}$. In other words, the vertex set of $T$ coincides with $V$ and vertices $v, v^{\prime} \in V$ are connected by an edge if and only if $Y_{v} \cap Y_{v^{\prime}} \neq \emptyset$, i.e., the edges of $T$ are associated with the walls. Clearly, $T$ is a tree with vertices of infinite (countable) degree. We equip $T$ with a length metric $\operatorname{dist}_{T}$ in which every edge has length 1.

LEMMA 4.1. If $A \subset Y_{v}, A^{\prime} \subset Y_{v^{\prime}}$ for some $A, A^{\prime} \in \mathcal{C}$ and $\operatorname{dist}_{T}\left(v, v^{\prime}\right)>1$ then $\operatorname{Hd}\left(A, A^{\prime}\right) \geq 10$.

Proof. The blocks $Y_{v}, Y_{v^{\prime}}$ are not adjacent by the condition. Thus each geodesic segment $a a^{\prime} \subset Y$ with $a \in A, a^{\prime} \in A^{\prime}$ must intersect at least two walls. Hence, $\operatorname{dist}\left(a, a^{\prime}\right) \geq 10$.

4.2. Exhaustion of $Y$ associated with a Busemann function on $T$. Fix $\xi \in \partial_{\infty} T$ and a Busemann function $B_{\xi}: T \rightarrow \mathbb{R}$ associated with $\xi$. We can assume that $B_{\xi}(v) \in \mathbb{Z}$ for each $v \in V$. Given $n \in \mathbb{Z}$, we define

$$
C_{n}=\cup\left\{Y_{v}: B_{\xi}(v) \leq n\right\}
$$

Then $C_{n}$ is a closed, convex subset in $Y$ whose boundary $\partial C_{n}$ is the countable union of walls. Clearly, we have $C_{n} \subset C_{n+1}$ and $Y=\cup_{n \in \mathbb{Z}} C_{n}$.

Furthermore, for each block $Y_{v}$ there is exactly one distinguished boundary component of $Y_{v}$, namely, the wall $W_{v}$ separating $Y_{v}$ from $\xi$. This wall corresponds to the first edge of the geodesic ray in $T$ from $v$ towards $\xi$.

4.3. $S H$-block structure on $Y$. We say that $Y$ has a $S H$-block structure, if the set $V$ can be decomposed as $V=S \cup H$ with the following properties: every block of type $S$ (Seifert type, or $S$-block for shortness) is isometric to the metric product, 
$Y_{v}=F_{v} \times \mathbb{R}$, where $F_{v} \subset \mathrm{H}^{2}$ is a convex subset bounded by countable many disjoint geodesic lines. Clearly, every $S$-block is a $\mathrm{CAT}(0)$ space.

Every block $Y_{v}$ of type $H$ (hyperbolic type, or $H$-block) is isometric to the complement of the union of countable many open disjoint horoballs in $\mathrm{H}^{3}$ (with the induced intrinsic metric). Such a block is also only a CAT(0) space because its boundary components are convex and flat.

Furthermore, we require that if different $S$-blocks $Y_{v}, Y_{v^{\prime}}$ are adjacent along a wall $W$, then the $\mathbb{R}$-factors of the decompositions $Y_{v}=F_{v} \times \mathbb{R}, Y_{v^{\prime}}=F_{v^{\prime}} \times \mathbb{R}$ are orthogonal along $W$ (we refer to this as the $\pi / 2$-condition).

We allow that any of the sets $S, H$ but not both might be empty.

We denote by Core $Y$ the union of all walls and $S$-blocks in $Y$. We do not exclude that Core $Y=Y$, however, we always have Core $Y \neq \emptyset$ by the definition of the $S H$ block structure. Each connected component of Core $Y$ is either a wall separating two $H$-blocks or the union of $S$-blocks and hence closed and convex. Furthermore, different components of Core $Y$ are separated by the distance at least 10 .

4.4. Existence of a $S H$-structure. In this section we show that a manifold satisfying the assumptions of Theorem B admits a $S H$-structure.

Decompose $M$ by the JSJ-decomposition into components which are Seifert fibered or atoroidal. Each component of the decomposition can be equipped with a structure modelled by the standard geometries, where the assumption rules out the $S^{3}$ and $S^{2} \times \mathbb{R}$ geometry. If the decomposition is nontrivial, only $\mathbb{R}^{3}, \mathrm{H}^{3}$ or $\mathrm{H}^{2} \times \mathbb{R}$ can occur, i.e. if one of the components is modelled by one of the remaining geometries NIL, SOL or $\widetilde{P S L}_{2}(\mathbb{R})$, then $M$ is a compact quotient of theses geometries.

Thus we can assume that the decomposition is nontrivial. Let us first assume that there is no hyperbolic piece in the decomposition. Then by a result of Kapovich and Leeb [KLe] the universal covering $Y$ of $M$ is bilipschitz to a manifold with $S H$ structure where all blocks are of type $S$. Note that in our definition of a block structure we have the assumption that walls are separated by 10 . This additional requirement is easily obtained.

If the decomposition contains a hyperbolic piece then by a result of Leeb [Le] $M$ carries a complete metric of nonpositive curvature. This metric is a geometric one on the Seifert pieces. On the hyperbolic pieces the metric is of constant curvature away from the boundary walls which is smoothly modified near the boundary tori and flat in a small neighbourhood of these tori. Using a bilipschitz modification in the way described by [KLe] for the metric on the universal covering $Y$ of $M$ one can assume that adjacent Seifert pieces satisfy the $\pi / 2$-condition and that different walls have distance $\geq 10$. In addition we assume that different walls of a hyperbolic piece have distance $\geq 12$, that the curvature is constant -1 outside of the 1-neighbourhood of the walls and $-1 \leq K \leq 0$ on the whole block. We refer to this metric as the smooth metric on $Y$. Near the boundary walls of hyperbolic pieces, the smooth metric does not satisfy the requirement of our definition of $H$-block. Thus we have to modify this metric near a wall to obtain a $S H$-block structure.

The 3-neighbourhood of a wall of a hyperbolic block in the smooth metric can be written as $F \times[0,3]$, where $F \times\{0\}$ is the boundary flat and the segments $t \mapsto\{p\} \times\{t\}$ are unit speed geodesics orthogonal to the boundary. On the other hand let $H \times[0, \infty)$ be the canonical parametrization of the closure of the complement of a horoball in $\mathrm{H}^{3}$. We will cut off $F \times[0,3]$ from $Y$ and glue back $H \times\left[0,2+t_{0}\right]$ where $t_{0}$ and isometries on both boundary components will be constructed in the sequel. The new metric will be bilipschitz to the old one and after the corresponding change at all walls of 
hyperbolic blocks we obtain a $S H$-structure.

Note that $\mathbb{Z}^{2}$ operates on $F \times[0,3]$. We choose an isometry $f_{0}: H \times\{0\} \rightarrow F \times\{0\}$ and obtain an isometric action of $\mathbb{Z}^{2}$ on $H \times[0, \infty)$. There exists a value $t_{0}$ such that the volumes of the tori $H \times\left\{t_{0}\right\} / \mathbb{Z}^{2}$ and $F \times\{1\} / \mathbb{Z}^{2}$ are equal. Note that $t_{0} \leq 1$ since $-1 \leq K \leq 0$ for the smooth metric. Consider the $\mathbb{Z}^{2}$-equivariant map $f_{1}: H \times\left\{t_{0}\right\} \rightarrow F \times\{1\}, f_{1}\left(p, t_{0}\right)=\left(f_{0}(p), 1\right)$. Let $f_{t}: H \times\left\{t \cdot t_{0}\right\} \rightarrow F \times\{t\}$ defined by $f_{t}\left(p, t \cdot t_{0}\right)=\left(f_{0}(p), t\right)$ for $0 \leq t \leq 1$. Now we deform $f_{1}$ in a $\mathbb{Z}^{2}$-invariant way via maps $f_{t}: H \times\left\{t_{0}-1+t\right\} \rightarrow F \times\{t\}, 1 \leq t \leq 2$, such that $f_{2}$ induces an affine map between the corresponding tori and such that all these maps preserve the volume. This is possible, since the curvature of the smooth metric is constant in this region. Finally we deform $f_{2}$ via maps $f_{t}: H \times\left\{t_{0}-1+t\right\} \rightarrow F \times\{t\}, 2 \leq t \leq 3$ to an isometry $f_{3}: H \times\left\{t_{0}+2\right\} \rightarrow F \times\{3\}$. In the last step we do not require the map to be equivariant. However it is clearly possible to choose this deformation in a way that we obtain a bilipschitz map $f: H \times\left[0,2+t_{0}\right] \rightarrow F \times[0,3]$ which is an isometry on both boundary components.

5. Proof of Theorem D. If the space $Y$ would be a CAT $(-1)$ space, it would be easy to construct a Lipschitz map $\mathcal{C} \rightarrow \mathcal{C}$ which associates to every $A \in \mathcal{C}$ a set with uniformly bounded diameter. E.g. choose a point $\omega \in \partial_{\infty} Y$ and take the convex projection of $A$ on the horoball centered at $\omega$ which lies distance 1 from $A$. One can then pick a point in this projected set to obtain a $(L, l)$-Lipschitz retraction $\mathcal{C} \rightarrow Y$.

In our situation we do not have this uniform hyperbolicity. Nevertheless we will construct a map Stop $\circ R_{0}: \mathcal{C} \rightarrow \mathcal{C}$ which associates to every $A \in \mathcal{C}$ a convex subset with a very special shape. The map $R_{0}: \mathcal{C} \rightarrow \mathcal{C}$ is a modification of the projection in the CAT $(-1)$-case and $R_{0}(A)$ has either small diameter or is contained in Core $Y$ (which was defined in sect. 4.3). The map $R_{0}$ is defined in sect. 5.2, 5.3.

The stopping map Stop $: \mathcal{C} \rightarrow \mathcal{C}$ is a general construction valid for any Hadamard space with a block structure. By the discussion in 4.2 a manifold $Y$ with a block structure has the exhaustion $Y=\cup_{n \in \mathbb{Z}} C_{n}$. Given this exhaustion one can associate to every $A \in \mathcal{C}$ a sequence $\left(A_{n}\right)_{n \in \mathbb{Z}}$ in $\mathcal{C}$ such that $A_{k}=A$ if $A \subset C_{k}$ and $A_{k} \subset C_{k}$. The construction of $A_{k}$ out of $A_{k+1}$ uses a geodesic in $\mathcal{C}$ associated to the distance function to $C_{k}$ as in sect. 3.2 .

In this way we associate to every $A \in \mathcal{C}$ a piecewise geodesic $\zeta_{A}$ and (in some sense) $\zeta_{A}$ depends Lipschitz on $A$ (see Lemma 5.1). The stopping map chooses an appropriate point on this piecewise geodesic. The result of Stop $\circ R_{0}$ is either

(i) a set with small diameter, or

(ii) up to a small error an interval in $\mathbb{R}$-direction of an $S$-block, or

(iii) essentially an arc on a circle in a boundary wall of a hyperbolic block.

In a last step we have to choose in each of these cases a point $R(A) \in Y$ in a consistent way. This choice is described in sect. 5.5.2.

The required quasi-Lipschitz retraction $R$ will be obtained as the composition of several admissible maps $\mathcal{C} \rightarrow \mathcal{C}$. A map $f: \mathcal{C} \rightarrow \mathcal{C}$ is said to be admissible if it decreases the diameter,

$$
\operatorname{diam} \circ f(A) \leq \operatorname{diam} A \text { for every } A \in \mathcal{C} .
$$

For instance, every convex projection is admissible by Lemma 3.2.

5.1. Stopping map Stop. In this section we assume that $Y$ has a blockstructure as defined in sect. $4.1,4.2$. 
5.1.1. Canonical piecewise geodesic paths in $\mathcal{C}$. We recall the exhaustion $Y=\cup_{n \in \mathbb{Z}} C_{n}$. Given $A \in \mathcal{C}, n \in \mathbb{Z}$, there is a unique path $\sigma_{A, n}:[0, \infty) \rightarrow \mathcal{C}$ associated with $C=C_{n}$ by Proposition 3.8. The restriction $\sigma_{A, n} \mid\left[0, t_{A, n}\right]$ is geodesic, where $t_{A, n}:=\inf \left\{t \geq 0: A \subset C_{n, t}\right\}$. Note that $t_{A, k}>0$ for every $k<n(A)$, where $n(A):=\min \left\{n \in \mathbb{Z}: A \subset C_{n}\right\}$.

For each $A \in \mathcal{C}$ we define the canonical piecewise geodesic path $\zeta_{A}:[0, \infty) \rightarrow \mathcal{C}$ using geodesics $\sigma_{A, n}$. A sequence of break points $t_{k} \geq 0, k \in \mathbb{Z}$ will be defined in such a way that $t_{k} \leq t_{k-1}, \zeta_{A} \mid\left[t_{k}, t_{k-1}\right]$ is a geodesic in $\mathcal{C}$ between $A_{k}=\zeta_{A}\left(t_{k}\right)$ and $A_{k-1}$, $A_{k} \subset C_{k}$ and $t_{k}$ is a minimal $t \geq 0$ for which $\zeta_{A}(t) \subset C_{k}$.

To this end, we put $n=n(\bar{A}), t_{k}=0, \zeta_{A}\left(t_{k}\right):=A=: A_{k}$ for all $k \geq n$. Assuming that $t_{k}, \zeta_{A} \mid\left[0, t_{k}\right]$ and $A_{k}=\zeta_{A}\left(t_{k}\right)$ are already defined for $k \leq n$, we put

$$
t_{k-1}=t_{k}+t_{A_{k}, k-1}, \quad A_{k-1}=\zeta_{A}\left(t_{k-1}\right), \quad \zeta_{A}(t)=\sigma_{A_{k}, k-1}\left(t_{k-1}-t\right),
$$

where $t_{k} \leq t \leq t_{k-1}$. This gives a well defined value $\zeta_{A}\left(t_{k}\right)$ since $\sigma_{A_{k}, k-1}\left(t_{A_{k}, k-1}\right)=$ $A_{k}$ by definitions of $\sigma_{A}$ and $t_{A}$. Furthermore, $\zeta_{k} \mid\left[t_{k}, t_{k-1}\right]$ is a unit speed geodesic in $\mathcal{C}$ between $A_{k}$ and $A_{k-1}$. For every $t \in\left(t_{k+1}, t_{k}\right]$, the set $\zeta_{A}(t) \subset Y$ lies in $C_{k, t_{k}-t}=\left\{y \in Y: \operatorname{dist}\left(y, C_{k}\right) \leq t_{k}-t\right\}$. Moreover, by Proposition 3.8, $\zeta_{A}(t)$ is the minimal convex subset in $C=C_{k, t_{k}-t}$ containing $\bar{p}_{C} \circ \zeta_{A}\left(t^{\prime}\right)$ for all $t_{k+1} \leq t^{\prime}<t$.

Finally, $\Delta_{k}(A):=t_{k-1}-t_{k}=t_{A_{k}, k-1} \geq 10$ for every $k<n(A)$ by the defining properties of our blocks and the definition of $t_{A}$. Hence, $\cup_{k \in \mathbb{Z}}\left[t_{k}, t_{k-1}\right]=[0, \infty)$, and $\zeta_{A}$ is defined on $[0, \infty)$.

It immediately follows from the definition and Lemma 3.2 that the map $A \mapsto \zeta_{A}(t)$ is admissible for every $t \geq 0$.

5.1.2. Monotonicity of the Hausdorff distance between canonical paths. Given $A, A^{\prime} \in \mathcal{C}$, we define continuous piecewise affine functions $s, s^{\prime}:[0, \infty) \rightarrow$ $[0, \infty), s(0)=0=s^{\prime}(0)$ depending both on $A, A^{\prime}$. We put inductively $t_{k-1}=$ $t_{k}+\max \left\{\Delta_{k}(A), \Delta_{k}\left(A^{\prime}\right)\right\}, k \in \mathbb{Z}$, subject to the condition $t_{k}=t_{k}\left(A, A^{\prime}\right)=0$ for each $k \geq \max \left\{n(A), n\left(A^{\prime}\right)\right\}$. Now, if $\Delta_{k}(A) \geq \Delta_{k}\left(A^{\prime}\right)$ then we define

$$
s\left(t_{k}+t\right)=t_{k}(A)+t \text { for } 0 \leq t \leq \Delta_{k}(A) .
$$

If $\Delta_{k}(A) \leq \Delta_{k}\left(A^{\prime}\right)$ then using $\Delta_{k}^{2}:=\Delta_{k}\left(A^{\prime}\right)-\Delta_{k}(A)$ we put

$$
s\left(t_{k}+t\right)= \begin{cases}t_{k}(A) & \text { for } 0 \leq t \leq \Delta_{k}^{2}, \\ t_{k}(A)+t-\Delta_{k}^{2} & \text { for } \Delta_{k}^{2} \leq t \leq \Delta_{k}\left(A^{\prime}\right) .\end{cases}
$$

Similarly, we define $s^{\prime}$ using break points $t_{k}\left(A^{\prime}\right)$ instead of $t_{k}(A)$.

The meaning of this definition is that the length $\Delta_{k}(A)$ of the geodesic subsegment $A_{k} A_{k-1} \subset \zeta_{A}$ might be larger than the corresponding length $\Delta_{k}\left(A^{\prime}\right)$ for $A^{\prime}$. In that case, we move along $\zeta_{A}$ by $s$ from $A_{k}$ to $A_{k-1}$ with unit speed simultaneously staying for a while at $A_{k}^{\prime}$ till $\zeta_{A} \circ s$ reaches the same level and then move along $\zeta_{A^{\prime}}$ by $s^{\prime}$ from $A_{k}^{\prime}$ to $A_{k-1}^{\prime}$ with unit speed.

Lemma 5.1. Given $A, A^{\prime} \in \mathcal{C}$, the function

$$
\psi_{A, A^{\prime}}(t)=\operatorname{Hd}\left(\zeta_{A} \circ s(t), \zeta_{A^{\prime}} \circ s^{\prime}(t)\right)
$$

decreases on $[0, \infty)$, where $s, s^{\prime}$ are defined as above.

Proof. This follows from Lemma 3.9 by untangling definitions of canonical paths $\zeta_{A}, \zeta_{A^{\prime}}$ and speeds $s, s^{\prime}$. 
Corollary 5.2. For every $A, A^{\prime} \in \mathcal{C}, k \in \mathbb{Z}$ we have

$$
\operatorname{Hd}\left(A_{k}, A_{k}^{\prime}\right) \leq \operatorname{Hd}\left(A, A^{\prime}\right)
$$

where $A_{k}=\zeta_{A} \circ t_{k}(A)$.

Proof. This follows from Lemma 5.1 and the fact that $t_{k}(A)=s\left(t_{k}\right), t_{k}\left(A^{\prime}\right)=$ $s^{\prime}\left(t_{k}\right)$, where $t_{k}=t_{k}\left(A, A^{\prime}\right) \in[0, \infty)$ is involved in the definition of the speeds $s, s^{\prime}$.

5.1.3. Critical, touching and stopping points of a canonical path. We denote by $\mathcal{C}_{1}$ the subspace of $\mathcal{C}$ which consists of elements sitting in one block. Clearly, $\mathcal{C}_{1}$ is closed in $\mathcal{C}$ and if a path $\zeta_{A}$ reaches $\mathcal{C}_{1}$ then it never leaves it. We define the critical point of $\zeta_{A}$ as the first point at which $\zeta_{A}$ hits $\mathcal{C}_{1}$,

$$
\mathrm{t}_{\mathrm{cr}}=\mathrm{t}_{\mathrm{cr}}(A)=\inf \left\{t \geq 0: \zeta_{A}(t) \in \mathcal{C}_{1}\right\}
$$

It follows from the definition of $\zeta_{A}$ that if $t \in\left[t_{k+1}, t_{k}\right]$ satisfies $t<t_{\mathrm{cr}}$ then $t_{k} \leq \mathrm{t}_{\mathrm{cr}}$. Thus $\mathrm{t}_{\mathrm{cr}}$ is always a break point for $\zeta_{A}, \mathrm{t}_{\mathrm{cr}}=t_{k}$ for some $k \in \mathbb{Z}$, or $\mathrm{t}_{\mathrm{cr}}=\infty$.

Lemma 5.3. For each $A \in \mathcal{C}$, the critical point $\mathrm{t}_{\mathrm{cr}}(A)$ is finite.

Proof. Since $A$ is bounded and distances between boundary components of all blocks are uniformly separated from 0 , there is only a finite number of blocks whose interiors intersect $A$ (if there is no such block then $A$ sits in a wall). If $t_{k}<\mathrm{t}_{\mathrm{cr}}$ for some $k<n(A)$ then passing through $t_{k}$ decreases this number for $\zeta_{A}(t)$ at least by 1 , whereas moving along $\zeta_{A}$ does not change this number while $t \in\left(t_{k}, t_{k-1}\right)$. Hence, the claim.

We denote by $A_{\mathrm{cr}}=\zeta_{A}\left(\mathrm{t}_{\mathrm{cr}}\right)$. One can easily predict the block where $A_{\mathrm{cr}}$ sits only by knowing $A \in \mathcal{C}$. To this end, consider the (finite) subtree $T_{A} \subset T$ spanned by the set $\left\{v \in V: A \cap \operatorname{Int} Y_{v} \neq \emptyset\right\}$ (if this set is empty then $A$ sits in a wall $W$ and $T_{A}$ is the edge of $T$ corresponding to $W$ ). There is a unique vertex $\bar{v} \in T_{A}$ with $B_{\xi}(\bar{v})=\min B_{\xi} \mid T_{A}=: k$. Then $A_{\mathrm{cr}} \subset Y_{\bar{v}}$ and, moreover, $A_{\mathrm{cr}} \cap A \neq \emptyset$ (however, it is not true in general that $\left.A_{\mathrm{cr}} \subset A\right)$. Everything interesting for us will take place either in $Y_{\bar{v}}$ or in the adjacent block $Y_{\bar{v}^{\prime}}$ with $B_{\xi}\left(\bar{v}^{\prime}\right)=k-1$. Namely, every point of the (broken) segment $\zeta_{A}\left(\left[t_{k}, \mu(A)\right]\right)$ with the stopping point $\mu(A)$ defined below corresponds to an element of $\mathcal{C}$ sitting in one of these two blocks. In particular, this holds for $\operatorname{Stop}(A)=\zeta_{A} \circ \mu(A)$.

The map crit $: \mathcal{C} \rightarrow \mathcal{C}_{1}, \operatorname{crit}(A)=A_{\text {cr }}$ is not continuous: every $A \in \mathcal{C}_{1}$ which touches $C_{n(A)-1}$ is a point of discontinuity, and jumps might be arbitrarily large. However, we have the following

Lemma 5.4. If $\operatorname{Hd}\left(A, A^{\prime}\right)<10$ for some $A, A^{\prime} \in \mathcal{C}$ then $A_{c r}, A_{c r}^{\prime}$ are sitting in one and the same block or in adjacent blocks.

Proof. We can assume that $A^{\prime}=A_{\mathrm{cr}}^{\prime}$ by Corollary 5.2. Next we note that $A \cap A_{\text {cr }} \neq \emptyset$ for each $A \in \mathcal{C}$. If the claim would not be true then for each $a \in A \cap A_{\text {cr }}$ we would have $\operatorname{dist}\left(a, A^{\prime}\right)=\operatorname{dist}\left(a, A_{\mathrm{cr}}^{\prime}\right) \geq 10$ since $A_{\mathrm{cr}}, A_{\mathrm{cr}}^{\prime}$ would be separated by at least one block. But then $\operatorname{Hd}\left(A, A^{\prime}\right) \geq 10$. This is a contradiction. $\square$

Assume that for $A \in \mathcal{C}$ we have $A_{k} \in \mathcal{C}_{1}$ for some $k \leq n(A)$. We define the $k$ th touching point $\tau_{k}=\tau_{k}(A)$ by $\tau_{k}:=t_{k-1}-\delta_{k}$, where

$$
\delta_{k}=\delta_{k}(A)=\inf \left\{\delta \geq 0: A_{k} \cap C_{k-1, \delta} \neq \emptyset\right\} .
$$

Obviously, $\delta_{k}(A) \leq \Delta_{k}(A)$, thus we have $\tau_{k} \in\left[t_{k}, t_{k-1}\right]$. Furthermore, $A_{k} \subset C_{k-1, \Delta_{k}} \backslash$ Int $C_{k-1, \delta_{k}}$ because $A_{k}$ sits in one block. Hence, $\tau_{k}-t_{k}=\Delta_{k}-\delta_{k} \leq \operatorname{diam} A_{k}$. 
Finally, we define the stopping point $\mu=\mu(A) \in[0, \infty)$ for $\zeta_{A}$ as follows. Let $\mathrm{t}_{\mathrm{cr}}=t_{k}$ be the critical point of $\zeta_{A}, k \leq n(A)$. We take the $k$ th touching point $\tau_{k} \in\left[t_{k}, t_{k-1}\right]$ and put $\mu=\tau_{k}+5$ if $\tau_{k}+5 \leq t_{k-1}$. Otherwise, we let $\lambda=5-\delta_{k}>0$ and put $\mu=\tau_{k-1}+\lambda$. So, we have $\mu \in\left[t_{k}, t_{k-1}\right]$ in the former case and we show that $\mu \in\left(t_{k-1}, t_{k-2}-5\right]$ in the last case (recall that $t_{k-2}-t_{k-1} \geq 10$ ). This is so because $t_{k-1} \leq \tau_{k-1}<\mu$ and $\lambda \leq 5<10 \leq \delta_{k-1}$. The inequality $\delta_{k-1} \geq 10$ holds for the following reason. Since $A_{k} \in \mathcal{C}_{1}$, the set $A_{k-1}$ sits in the wall $W_{v}$ of the block $Y_{v}$ containing $A_{k}, B_{\xi}(v)=k$. Then the level $\delta_{k-1}$ is at least the minimal distance between different boundary components of the blocks, i.e., $\delta_{k-1} \geq 10$. Hence, the claim.

We define a map Stop $: \mathcal{C} \rightarrow \mathcal{C}$ by $\operatorname{Stop}(A)=\zeta_{A}$ o $\mu(A)$. We also put $\delta(A):=\delta_{k}(A)$, where $t_{k}=\mathrm{t}_{\mathrm{cr}}$ is the critical point of $\zeta_{A}, k \leq n(A)$. Again, Stop possesses points of discontinuity. Nevertheless, we have the following

Proposition 5.5. For every $A, A^{\prime} \in \mathcal{C}$ with $H:=\operatorname{Hd}\left(A, A^{\prime}\right)<5$ the sets $\operatorname{Stop}(A), \operatorname{Stop}\left(A^{\prime}\right)$ sit in one and the same block and

$$
\operatorname{Hd}\left(\operatorname{Stop}(A), \operatorname{Stop}\left(A^{\prime}\right)\right) \leq 3 H
$$

except may be the case when $A_{c r}, A_{c r}^{\prime}$ sit in one and the same block and $\delta(A) \geq 5>$ $\delta\left(A^{\prime}\right)$. In this exceptional case we have

$$
\operatorname{Hd}\left(\operatorname{Stop}(A), \operatorname{Stop}\left(A^{\prime}\right)\right) \leq 4 H+\operatorname{diam} \circ \operatorname{Stop}(A) .
$$

Furthermore, $\operatorname{Stop}(y) \in Y$ for every $y \in Y \subset \mathcal{C}$ and $\operatorname{dist}(y, \operatorname{Stop}(y)) \leq 5$.

Proof. We consider several cases.

(a) $A_{\mathrm{cr}}, A_{\mathrm{cr}}^{\prime}$ sit in different blocks. Using Lemma 5.4, we can assume W.L.G. that $A_{\mathrm{cr}}=A_{k}, A_{\mathrm{cr}}^{\prime}=A_{k-1}^{\prime}$. By Corollary 5.2, $\operatorname{Hd}\left(A_{k}^{\prime}, A_{k}\right) \leq H$. Next we have either $A_{k}^{\prime}=A_{k-1}^{\prime}=A^{\prime}$ or $A_{k}^{\prime} \notin \mathcal{C}_{1}$. In either case, $A_{k}^{\prime}$ meets $C_{k-1}$. Thus $\operatorname{Hd}\left(A_{k}^{\prime}, A_{k}\right) \geq \delta(A)$ because $A_{k}$ does not intersect Int $C_{k-1, \delta(A)}$. Hence, $\delta(A) \leq H<5$. This means that both stopping points $\operatorname{Stop}(A), \operatorname{Stop}\left(A^{\prime}\right)$ sit in one and the same block $Y_{v^{\prime}}, B_{\xi}\left(v^{\prime}\right)=$ $k-1$ and $\mu(A)=t_{k-2}-\rho, \mu\left(A^{\prime}\right)=t_{k-2}^{\prime}-\rho^{\prime}$, where $\rho=\delta_{k-1}+\delta(A)-5, \rho^{\prime}=\delta_{k-1}^{\prime}-5$. Note that $\left|\delta_{k-1}-\delta_{k-1}^{\prime}\right| \leq \operatorname{Hd}\left(A_{k-1}, A_{k-1}^{\prime}\right) \leq H$ and

$$
\left|\rho-\rho^{\prime}\right| \leq\left|\delta_{k-1}-\delta_{k-1}^{\prime}\right|+\delta(A) \leq 2 H \text {. }
$$

By Lemma 5.1, we have

$$
\operatorname{Hd}\left(\zeta_{A}\left(t_{k-2}-s\right), \zeta_{A^{\prime}}\left(t_{k-2}^{\prime}-s\right)\right) \leq H
$$

for every $s, 0 \leq s \leq \min \left\{\Delta_{k-1}(A), \Delta_{k-1}\left(A^{\prime}\right)\right\}$. Since $\rho \leq \Delta_{k-1}(A), \rho^{\prime} \leq \Delta_{k-1}\left(A^{\prime}\right)$, we obtain

$$
\begin{aligned}
\operatorname{Hd}\left(\operatorname{Stop}(A), \operatorname{Stop}\left(A^{\prime}\right)\right) & =\operatorname{Hd}\left(\zeta_{A}\left(t_{k-2}-\rho\right), \zeta_{A^{\prime}}\left(t_{k-2}^{\prime}-\rho^{\prime}\right)\right) \\
& \leq \operatorname{Hd}\left(\zeta_{A}\left(t_{k-2}-\rho\right), \zeta_{A^{\prime}}\left(t_{k-2}^{\prime}-\rho\right)\right)+\left|\rho-\rho^{\prime}\right| \\
& \leq 3 H .
\end{aligned}
$$

(b) $A_{\mathrm{cr}}, A_{\mathrm{cr}}^{\prime}$ sit in one and the same block $Y_{v}$ and $\delta(A), \delta\left(A^{\prime}\right) \geq 5$ or $\delta(A), \delta\left(A^{\prime}\right)<$ 5. We have $A_{\mathrm{cr}}=A_{k}, A_{\mathrm{cr}}^{\prime}=A_{k}^{\prime}$ for $k=B_{\xi}(v)$ and $\left|\delta(A)-\delta\left(A^{\prime}\right)\right| \leq H$. Suppose first that $\delta(A), \delta\left(A^{\prime}\right) \geq 5$. This means that both stopping points $\operatorname{Stop}(A), \operatorname{Stop}\left(A^{\prime}\right)$ sit in 
$Y_{v}$ and $\mu(A)=t_{k-1}-\rho, \mu\left(A^{\prime}\right)=t_{k-1}^{\prime}-\rho^{\prime}$ for $\rho=\delta(A)-5, \rho^{\prime}=\delta\left(A^{\prime}\right)-5$. As above, we obtain

$$
\operatorname{Hd}\left(\operatorname{Stop}(A), \operatorname{Stop}\left(A^{\prime}\right)\right) \leq \operatorname{Hd}\left(\zeta_{A}\left(t_{k-2}-\rho\right), \zeta_{A^{\prime}}\left(t_{k-2}^{\prime}-\rho\right)\right)+\left|\rho-\rho^{\prime}\right| \leq 2 H .
$$

Now we assume that $\delta(A), \delta\left(A^{\prime}\right)<5$. As in the case (a), this means that both stopping points $\operatorname{Stop}(A), \operatorname{Stop}\left(A^{\prime}\right)$ sit in $Y_{v^{\prime}}$ adjacent to $Y_{v}, B_{\xi}\left(v^{\prime}\right)=k-1$. In this case, we have $\mu(A)=t_{k-2}-\rho, \mu\left(A^{\prime}\right)=t_{k-2}^{\prime}-\rho^{\prime}$ for $\rho=\delta_{k-1}+\delta(A)-5, \rho^{\prime}=\delta_{k-1}^{\prime}+\delta\left(A^{\prime}\right)-5$ and $\left|\rho-\rho^{\prime}\right| \leq\left|\delta_{k-1}-\delta_{k-1}^{\prime}\right|+\left|\delta(A)-\delta\left(A^{\prime}\right)\right| \leq 2 H$. As in the case (a), this implies

$$
\operatorname{Hd}\left(\operatorname{Stop}(A), \operatorname{Stop}\left(A^{\prime}\right)\right) \leq 3 H \text {. }
$$

(c) $A_{\mathrm{cr}}, A_{\mathrm{cr}}^{\prime}$ sit in $Y_{v}$ and $\delta(A) \geq 5>\delta\left(A^{\prime}\right)$. We have $0<\delta(A)-\delta\left(A^{\prime}\right) \leq H$ and $\operatorname{Hd}\left(\operatorname{Stop}(A), \zeta_{A^{\prime}}\left(t_{k-1}^{\prime}-\rho\right)\right)=\operatorname{Hd}\left(\zeta_{A}\left(t_{k-1}-\rho\right), \zeta_{A^{\prime}}\left(t_{k-1}^{\prime}-\rho\right)\right) \leq H$ for $\rho=$ $\delta(A)-5$ by Lemma 5.1. The Hausdorff distance between $\zeta_{A^{\prime}}\left(t_{k-1}^{\prime}-\rho\right)$ and $\operatorname{Stop}\left(A^{\prime}\right)=$ $\zeta_{A^{\prime}} \circ \mu\left(A^{\prime}\right)$ can be estimated from above as the sum of lengths of three geodesic segments in $\mathcal{C}$ obtained by restricting $\zeta_{A^{\prime}}$ on $\left[t_{k-1}^{\prime}-\rho, t_{k-1}^{\prime}\right],\left[t_{k-1}^{\prime}, \tau_{k-1}^{\prime}\right],\left[\tau_{k-1}^{\prime}, \mu\left(A^{\prime}\right)\right]$ respectively. The length of the first one is $\rho$, the second one at most diam $A_{k-1}^{\prime}$ and the last one $5-\delta\left(A^{\prime}\right)$. All together they give at most $\delta(A)-\delta\left(A^{\prime}\right)+\operatorname{diam} A_{k-1}^{\prime}$. Since $\operatorname{Hd}\left(A_{k-1}, A_{k-1}^{\prime}\right) \leq H$ and $\operatorname{diam} A_{k-1} \leq \operatorname{diam} \circ \operatorname{Stop}(A)$, we have $\operatorname{diam} A_{k-1}^{\prime} \leq$ $\operatorname{diam} \circ \operatorname{Stop}(A)+2 H$. Thus

$$
\operatorname{Hd}\left(\operatorname{Stop}(A), \operatorname{Stop}\left(A^{\prime}\right)\right) \leq 4 H+\operatorname{diam} \circ \operatorname{Stop}(A) .
$$

The last assertion of the Proposition immediately follows from the definition of Stop.

From Proposition 5.5 we easily obtain

Corollary 5.6. For any choice $R(A) \in \operatorname{Stop}(A)$ we have

$$
\operatorname{dist}\left(R(A), R\left(A^{\prime}\right)\right) \leq 4 \mathrm{Hd}\left(A, A^{\prime}\right)+2 D
$$

for every $A, A^{\prime} \in \mathcal{C}$ with $\operatorname{diam} \circ \operatorname{Stop}(A) \leq D$ and $\operatorname{diam} \circ \operatorname{Stop}\left(A^{\prime}\right) \leq D$, and $\operatorname{dist}(y, R(y)) \leq 5$ for every $y \in Y \subset \mathcal{C}$.

This Corollary shows that to prove Theorem $\mathrm{D}$ we have to make a good choice $R(A) \in \operatorname{Stop}(A)$ for stopping sets with large diameter. To this end, we make a preliminary step which is needed only in the case when there are hyperbolic blocks in our $S H$-block structure on $Y$. This step is described in the following two sections.

5.2. Diameter projection associated with Busemann function. In section 5.2 and 5.3 we construct the map $R_{0}: \mathcal{C} \rightarrow \mathcal{C}$. Here we consider an arbitrary Hadamard space $Y$ and the associated $\mathcal{C}=\mathcal{C}(Y)$.

Given $\omega \in \partial_{\infty} Y$, we consider a Busemann function $b_{\omega}: Y \rightarrow \mathbb{R}$ associated with $\omega$. Its sublevel sets $C_{t}=\left\{y \in Y: b_{\omega}(y) \leq t\right\}$ are convex, thus the metric projections $p_{t}: Y \rightarrow C_{t}, t \in \mathbb{R}$ are 1-Lipschitz. We fix $\Lambda>0$ and for $A \in \mathcal{C}$ put

$$
t(A):=\min b_{\omega} \mid A-\Lambda \cdot \operatorname{diam} A .
$$

Now, we define $p_{\omega, \Lambda}: \mathcal{C} \rightarrow \mathcal{C}$ by $p_{\omega, \Lambda}(A)=$ conv o $p_{t(A)}(A)$. Obviously, $p_{\omega, \Lambda}(y)=y$ for every $y \in Y \subset \mathcal{C}$.

LEMMA 5.7. The map $p_{\omega, \Lambda}$ is $2(\Lambda+1)$-Lipschitz. 
Proof. Given $A, A^{\prime} \in \mathcal{C}$, we denote $\widehat{A}=p_{\omega, \Lambda}(A), \widehat{A}^{\prime}=p_{\omega, \Lambda}\left(A^{\prime}\right), t=t(A)$, $t^{\prime}=t\left(A^{\prime}\right)$ and W.L.G. assume that $t^{\prime} \leq t$. Then

$$
\begin{aligned}
\operatorname{Hd}\left(\widehat{A}, \widehat{A}^{\prime}\right) & \leq \operatorname{Hd}\left(p_{t}(A), p_{t^{\prime}}\left(A^{\prime}\right)\right) \quad \text { by Lemma } 3.1 \\
& \leq \operatorname{Hd}\left(p_{t}(A), p_{t}\left(A^{\prime}\right)\right)+t-t^{\prime}
\end{aligned}
$$

because $p_{t^{\prime}}\left(A^{\prime}\right)=p_{t^{\prime}} \circ p_{t}\left(A^{\prime}\right)$ and $\mathrm{Hd}\left(p_{t}\left(A^{\prime}\right), p_{t^{\prime}}\left(A^{\prime}\right)\right) \leq t-t^{\prime}$. Furthermore, $\operatorname{Hd}\left(p_{t}(A), p_{t}\left(A^{\prime}\right)\right) \leq \operatorname{Hd}\left(A, A^{\prime}\right)$ because $p_{t}$ is 1-Lipschitz. Clearly, $\left|d-d^{\prime}\right| \leq \operatorname{Hd}\left(A, A^{\prime}\right)$ for $d=\min b_{\omega}\left|A, \bar{d}^{\prime}=\min b_{\omega}\right| A^{\prime}$ and

$$
\left|t-t^{\prime}\right| \leq\left|d-d^{\prime}\right|+\Lambda\left|\operatorname{diam} A-\operatorname{diam} A^{\prime}\right|
$$

Using Lemma 3.3 , we obtain $\left|t-t^{\prime}\right| \leq(2 \Lambda+1) \operatorname{Hd}\left(A, A^{\prime}\right)$ and hence

$$
\operatorname{Hd}\left(\widehat{A}, \widehat{A}^{\prime}\right) \leq 2(\Lambda+1) \operatorname{Hd}\left(A, A^{\prime}\right)
$$

We fix $\varepsilon>0$ and consider the set $Y_{-1}(\varepsilon)$ consisting of all $y \in Y$ such that the ball $B_{2 \varepsilon}(y) \subset Y$ is a $\mathrm{CAT}(-1)$ space. Next, we let $a a^{\prime} \subset \mathrm{H}^{2}$ be a segment of length $\varepsilon$; segments $a b, a^{\prime} b^{\prime}$ are orthogonal to $a a^{\prime}$ at $a, a^{\prime}$ respectively, have lengths $\varepsilon$ and lie in one and the same half-plane w.r.t. the geodesic in $\mathrm{H}^{2}$ extending $a a^{\prime}$. Now, we put

$$
\Lambda=\Lambda_{\varepsilon}:=\max \left\{1,2 \varepsilon /\left(\operatorname{dist}\left(b, b^{\prime}\right)-\varepsilon\right)\right\} .
$$

Note that $\Lambda_{\varepsilon} \rightarrow \infty$ as $\varepsilon \rightarrow 0$.

Proposition 5.8. For each $A \in \mathcal{C}$, we have either diam $\circ p_{\omega, \Lambda}(A)<\varepsilon$ or

$$
p_{t(A)}(A) \cap Y_{-1}(\varepsilon)=\emptyset \text {. }
$$

Proof. Assume that diam $\circ p_{\omega, \Lambda}(A) \geq \varepsilon$ and $\widetilde{A} \cap Y_{-1}(\varepsilon) \neq \emptyset$ for some $A \in \mathcal{C}$, where $\widetilde{A}=p_{t(A)}(A)$. Note that $\operatorname{diam} \widetilde{A} \geq \varepsilon$ by Lemma $3.1, \widetilde{A} \subset \partial C_{t(A)}$ by the definition of $t(A)$, and $\widetilde{A}$ is connected, because $A$ is connected.

By the assumption, there exists $a \in \widetilde{A} \cap Y_{-1}(\varepsilon)$. Hence, we can find $a^{\prime} \in \widetilde{A}$ with $\operatorname{dist}\left(a, a^{\prime}\right)=\varepsilon$ and $c, c^{\prime} \in A$ with $p_{t(A)}(c)=a, p_{t(A)}\left(c^{\prime}\right)=a^{\prime}$. For $t \in[0, \Lambda \operatorname{diam} A]$, we define $a(t) \in a c, a^{\prime}(t) \in a^{\prime} c^{\prime}$ by $\operatorname{dist}(a(t), a)=t=\operatorname{dist}\left(a^{\prime}(t), a^{\prime}\right)$. Note that $\operatorname{diam} A \geq$ $\operatorname{diam} \widetilde{A} \geq \varepsilon$, thus $\varepsilon \in[0, \Lambda \operatorname{diam} A]$. Then the function $L(t)=\operatorname{dist}\left(a(t), a^{\prime}(t)\right)$ is convex and increasing, thus $\operatorname{dist}\left(c, c^{\prime}\right) \geq L(\Lambda \operatorname{diam} A) \geq L(\varepsilon)+L^{\prime}(\varepsilon)(\Lambda \operatorname{diam} A-\varepsilon)$, where $L^{\prime}(\varepsilon)$ is the local Lipschitz constant for $L$ at $t=\varepsilon$. On the other hand, the quadrangle $a(\varepsilon) a a^{\prime} a^{\prime}(\varepsilon)$ is contained in the ball $B_{2 \varepsilon}(a)$ which is a CAT $(-1)$ space. Comparison with $\mathrm{H}^{2}$ and the definition of $\Lambda$ imply that $L^{\prime}(\varepsilon) \cdot \Lambda \geq 2$. Hence,

$$
\operatorname{dist}\left(c, c^{\prime}\right)>2 \operatorname{diam} A-\varepsilon \geq \operatorname{diam} A,
$$

since $L(\varepsilon)>L(0)=\varepsilon$ and $L^{\prime}(\varepsilon) \leq 2$. This is a contradiction, because $c, c^{\prime} \in A$. $\square$

5.3. Projecting on Core $Y$. Here we come back to a Hadamard space $Y$ with $S H$-block structure. We fix $\varepsilon \in(0,1]$ and consider the set $Y_{-1}(\varepsilon)$ introduced in 5.2 . In $S H$-block structure case we, obviously, have

$$
\begin{aligned}
Y_{-1}(\varepsilon) & =\{y \in Y: \operatorname{dist}(y, \operatorname{Core} Y) \geq 2 \varepsilon\} \\
& =\bigcup_{v \in H}\left\{y \in Y_{v}: \operatorname{dist}\left(y, \partial Y_{v}\right) \geq 2 \varepsilon\right\} .
\end{aligned}
$$


Let $\mathcal{C}_{0} \subset \mathcal{C}$ be the subset of all $A \in \mathcal{C}$ such that either $\operatorname{diam} A \leq 1$ or $A \subset$ Core $Y$.

Lemma 5.9. There exists an admissible $(L, l)$-Lipschitz map $R_{0}: \mathcal{C} \rightarrow \mathcal{C}_{0}$ with $L=L(\varepsilon)>0, l=4 \varepsilon$ such that $R_{0}(y)=y$ for each $y \in Y$. Here $L(\varepsilon) \rightarrow \infty$ as $\varepsilon \rightarrow 0$.

Proof. We fix $\omega \in \partial_{\infty} Y$ and define $\Lambda=\Lambda_{\varepsilon}$ as in Proposition 5.8. Then for each $A \in \mathcal{C}$ we have either $\operatorname{diam} \widetilde{A}<\varepsilon$ or $\widetilde{A}:=p_{\omega, \Lambda}(A)$ lies in the $2 \varepsilon$-neighbourhood of a component of Core $Y$. The last conclusion follows from Proposition 5.8 and the fact that the $2 \varepsilon$-neighbourhood of Core $Y$ in $Y$ is the union of convex sets pairwise separated by the distance at least $10-4 \varepsilon \geq 6$. Hence, if diam $\widetilde{A} \geq 1$ then the convex projection

$$
\bar{p}_{\text {Core } Y}(\widetilde{A})=\operatorname{conv} o p_{\operatorname{Core} Y}(\widetilde{A}) \subset \operatorname{Core} Y
$$

is well defined and $\operatorname{Hd}\left(\widetilde{A}, \bar{p}_{\text {Core } Y}(\widetilde{A})\right) \leq 2 \varepsilon$.

By Lemma 5.7, the admissible map $p_{\omega, \Lambda}: \mathcal{C} \rightarrow \mathcal{C}$ is $L$-Lipschitz with $L=2(\Lambda+1)$. Furthermore, $p_{\omega, \Lambda}(y)=y$ for each $y \in Y$ (see sect. 5.2). Now, we define $R_{0}: \mathcal{C} \rightarrow \mathcal{C}$ by

$$
R_{0}(A)=\bar{p}_{\text {Core } Y} \circ p_{\omega, \Lambda}(A) \quad \text { if } \quad \operatorname{diam} \circ p_{\omega, \Lambda}(A) \geq 1
$$

and $R_{0}(A)=p_{\omega, \Lambda}(A)$ otherwise. Then $R_{0}$ is admissible, $R_{0}(\mathcal{C}) \subset \mathcal{C}_{0}, R_{0}(y)=y$ for each $y \in Y$. Furthermore, for $\widetilde{A}=p_{\omega, \Lambda}(A), \widetilde{A}^{\prime}=p_{\omega, \Lambda}\left(A^{\prime}\right)$ we have $\operatorname{Hd}\left(\widetilde{A}, \widetilde{A}^{\prime}\right) \leq$ $L \cdot \operatorname{Hd}\left(A, A^{\prime}\right) ; \operatorname{Hd}\left(R_{0}(A), \widetilde{A}\right), \operatorname{Hd}\left(R_{0}\left(A^{\prime}\right), \widetilde{A}^{\prime}\right) \leq 2 \varepsilon$. Thus

$$
\operatorname{Hd}\left(R_{0}(A), R_{0}\left(A^{\prime}\right)\right) \leq L \cdot \operatorname{Hd}\left(A, A^{\prime}\right)+4 \varepsilon
$$

i.e., $R_{0}$ is $(L, l)$-Lipschitz with $l=4 \varepsilon$ and $L=2(\Lambda+1) \rightarrow \infty$ as $\varepsilon \rightarrow 0$.

5.4. Stopping sets with large diameter. Here we study the case that $A \in \mathcal{C}_{0}$ and $\operatorname{Stop}(A)$ has a large diameter.

5.4.1. Sitting in an $H$-block. Let $Y_{v}$ be an $H$-block, $W_{v} \subset Y_{v}$ the distinguished wall (see sect. 4.2), $W \subset Y_{v}$ a wall different from $W_{v}$. We define a subset $K \subset W$ by the condition $x \in K$ if and only if the geodesic segment $x p_{v}(x)$ is transversal to $W$ at $x$, where $p_{v}: Y \rightarrow W_{v}$ is the metric projection. Clearly, $p_{v}$ restricted on $Y_{v}$ coincides with $p_{C_{k}}$ restricted on $Y_{v}, k=B_{\xi}(v)$. The meaning of this definition is that if $x \in W \backslash \bar{K}$ then moving along $x p_{v}(x)$ from $x$ to $p_{v}(x)$ one first goes along $W$ until meets $\bar{K}$ and only then one leaves $W$ towards $W_{v}$.

We put $W(\rho)=W \cap C_{k-1, \rho}$ which evidently coincides with $\{x \in W$ : $\left.\operatorname{dist}\left(x, W_{v}\right) \leq \rho\right\}$. We also let $\rho_{0}=\inf \{\rho \geq 0: \bar{K} \subset W(\rho)\}$. It is convenient to use notation $B_{r}^{W}(x)$ for a closed ball in $W$ of radius $r$ centered at $x$.

Obviously, there exists a unique point $x_{0} \in W$ with $\operatorname{dist}\left(x_{0}, p_{v}\left(x_{0}\right)\right)=$ $\operatorname{dist}\left(W, W_{v}\right)=: \rho_{v}$.

LEMMA 5.10. We have $\bar{K} \subset W\left(\rho_{0}\right) \subset B_{r_{0}}^{W}\left(x_{0}\right)$ for some $r_{0}<2$.

Proof. Consider a horocycle $S \subset \mathrm{H}^{2}$ and a geodesic line $\gamma \subset \mathrm{H}^{2}$ which touches $S$ at $x \in S$. Then $p_{\gamma}(S) \subset \gamma$ is a subsegment of length $2 r_{1}$ with $r_{1}=-\ln \tan \frac{\pi}{8}=0.98 \ldots$ centered at $x$ as an easy computation in hyperbolic geometry shows. Moreover, $p_{\gamma}(S)=p_{\gamma}\left(\left[s s^{\prime}\right]\right)$ where $\left[s s^{\prime}\right] \subset S$ is a subsegment of length $2 r_{2}$ centered at $x$ with $r_{2}=\sqrt{2}$, and $\operatorname{dist}(\gamma, s)=\operatorname{dist}\left(\gamma, s^{\prime}\right)=r_{1}$. It follows that $\bar{K} \subset B_{r_{2}}^{W}\left(x_{0}\right)$ by comparison with $\mathrm{H}^{3}$ because the other boundary walls of $Y_{v}$ are far away to intervene. Their influence is only that we cannot say that $\partial C_{k-1, \rho}$ has the shape of a horosphere in 
$\mathrm{H}^{3}$, however, we know that $C_{k-1, \rho}$ is convex. Thus considering everything in the ball $B_{10}\left(x_{0}\right) \subset Y_{v}$ we see that $C_{k-1, \rho_{v}} \cap B_{10}\left(x_{0}\right)$ is contained in a half-space bounded by the hyperbolic 2-plane $M$ which touches $W$ at $x_{0}$. Next we have $\rho_{0}-\rho_{v} \leq r_{2}$ because the maximal distance from points of $\bar{K}$ to $C_{k-1, \rho_{v}}$ is at most that to $x_{0}$. Note that the distance between $\partial B_{r_{2}}^{W}\left(x_{0}\right)$ and $M$ in $Y_{v}$ is equal to $r_{1}$ by the consideration above with the horocycle $S \subset \mathrm{H}^{2}$. It follows that $W\left(\rho_{0}\right)=W \cap C_{k-1, \rho_{v}}+\left(\rho_{0}-\rho_{v}\right)$ is contained in $B_{r_{0}}^{W}\left(x_{0}\right)$ with $r_{0}=r_{2}+\left(\rho_{0}-\rho_{v}-r_{1}\right) \leq 2 r_{2}-r_{1}<2$.

The following Proposition describes the shape of stopping sets with large diameter sitting in an $H$-block.

Proposition 5.11. If $\operatorname{diam} \circ \operatorname{Stop}(A) \geq 4$ and $\operatorname{Stop}(A)$ sits in an $H$-block $Y_{v}$ for some $A \in \mathcal{C}_{0}$ then $\zeta_{A}(t) \subset W$ for all $t \in\left[t_{k}, \mu(A)\right]$, where $W$ is a wall of $Y_{v}$ different from $W_{v}, k=B_{\xi}(v)$. In particular, $\operatorname{Stop}(A) \subset W$. Furthermore, $\zeta_{A}(t)$ is the convex hull in $W$ of an arc on the boundary $\partial W(\rho)$ with $\rho=t_{k-1}-t \geq \rho_{0}$ (note that $W(\rho)$ is convex and $\partial W(\rho)$ equidistant to $\partial W\left(\rho_{0}\right)$ in $\left.W\right)$.

Proof. We have diam $A \geq \operatorname{diam} \circ \operatorname{Stop}(A)>1$, hence $A \subset$ Core $Y$. It implies $A_{k} \subset W$ for some wall $W \subset \partial Y_{v}$ different from $W_{v}$. Since $\bar{K} \subset W\left(\rho_{0}\right)$, we have $\zeta_{A}(t) \subset W$ for each $t \in\left[t_{k}, t_{k-1}-\rho_{0}\right]$. This follows from definitions of $\zeta_{A}$ and $K$. It suffices to show that $\mu(A) \leq t_{k-1}-\rho_{0}$. The assumption $\mu(A)>t_{k-1}-\rho_{0}$ would imply

$$
\operatorname{Stop}(A)=\zeta_{A} \circ \mu(A) \subset W\left(\rho_{0}\right) \subset B_{r_{0}}^{W}\left(x_{0}\right)
$$

by Lemma 5.10. Thus $\operatorname{diam} \circ \operatorname{Stop}(A) \leq 2 r_{0}<4$ which is a contradiction.

\subsubsection{Sitting in an $S$-block. We need the following}

Lemma 5.12. Assume that $\operatorname{diam} A>1$ and $A_{c r}$ sits in an $H$-block $Y_{v}$ for some $A \in \mathcal{C}_{0}$. Then $A=A_{c r} \subset \partial Y_{v}$ and $\delta(A) \geq 10$.

Proof. We have $A \subset$ Core $Y$ according to the definition of $\mathcal{C}_{0}$. Then $A_{\mathrm{cr}} \subset$ $Y_{v} \cap \operatorname{Core} Y \subset \partial Y_{v}$ since $Y_{v}$ is an $H$-block. Thus $A_{\text {cr }}$ being connected sits in a boundary wall $W$ of $Y_{v}$. It follows that $A \subset W$ since otherwise $A$ would intersect the interior of Core $Y$ and consequently $A_{\mathrm{cr}}$ could not be a subset of $Y_{v}$. Hence, $A_{\text {cr }}=A$. The wall $W$ is different from the distinguished wall $W_{v} \subset \partial Y_{v}$. Thus $\delta(A) \geq \operatorname{dist}\left(W, W_{v}\right) \geq 10$.

Let $Y_{v}$ be an $S$-block. For $A \subset Y_{v}$ we denote by $A_{\mathrm{H}^{2}}$ the projection of $A$ on the $\mathrm{H}^{2}$-factor $F_{v}$ and by $A_{\mathbb{R}}$ the projection of $A$ on the $\mathbb{R}$-factor of the decomposition $Y_{v}=F_{v} \times \mathbb{R}$.

Now we describe the shape of large stopping sets sitting in an $S$-block.

Proposition 5.13. Assume that $\operatorname{diam} \circ \operatorname{Stop}(-A) \geq 4$ and $\operatorname{Stop}(A)$ sits in an $S$-block $Y_{v}$ for some $A \in \mathcal{C}_{0}$. Then $\operatorname{diam}(\operatorname{Stop}(A))_{\mathrm{H}^{2}} \leq 0.1$ and moreover either $\delta(A) \geq 5$ or $\delta(A)<1$.

Proof. If $\delta(A) \geq 5$ then $A_{\mathrm{cr}} \subset Y_{v}$ and dist $\left._{\mathrm{H}^{2}}\left(\left(A_{\mathrm{cr}}\right)_{\mathrm{H}^{2}} \text {, (Stop } A\right)_{\mathrm{H}^{2}}\right) \geq 5$. Thus $\operatorname{diam}(\operatorname{Stop}(A))_{\mathrm{H}^{2}} \leq 2 \ln \frac{e^{5}+1}{e^{5}-1}<0.04$ as an easy computation in hyperbolic geometry shows.

Assume now that $\delta(A)<1$. This means that $\zeta_{A}(t)$ moves along $\zeta_{A}$ by the distance $5-\delta(A)>4$ between the corresponding touching point and $\operatorname{Stop}(A)$. Thus again diam $(\operatorname{Stop}(A))_{\mathrm{H}^{2}} \leq 2 \ln \frac{e^{4}+1}{e^{4}-1}<0.08$.

Finally, assume that $1 \leq \delta(A)<5$. We have $A_{\mathrm{cr}} \subset Y_{v^{\prime}}$ where the block $Y_{v^{\prime}}$ is adjacent to $Y_{v}, B_{\xi}\left(v^{\prime}\right)=k+1$ for $k=B_{\xi}(v)$, and $\operatorname{diam} A \geq \operatorname{diam} \circ \operatorname{Stop}(A)>1$. It follows that $Y_{v^{\prime}}$ is an $S$-block, since otherwise $\delta(A) \geq 10$ by Lemma 5.12. Moving 
at least by 1 in $Y_{v^{\prime}}$ along $\zeta_{A}$ shrinks $\zeta_{A}(t)$ to the size at most $2 \ln \frac{e+1}{e-1}<1.6$ in the $\mathrm{H}^{2}$-direction of $Y_{v^{\prime}}$. To reach $\operatorname{Stop}(A)$ one has first to project $A_{k+1}$ on $C_{k, \delta}$ with $\delta \geq 10$, which is the minimal distance between boundary components of $Y_{v}$. This shrinks $\zeta_{A}(t)$ to the size at most $2 \frac{e^{10}+1}{e^{10}-1}<2.1$ in the $\mathrm{H}^{2}$-direction of $Y_{v}$ by an easy computation in hyperbolic geometry. However, the $\mathrm{H}^{2}$-directions for $Y_{v^{\prime}}$ and $Y_{v}$ are mutually orthogonal along the separating wall by the $\pi / 2$-condition (see sect. 4.3 ). Hence, diam $\circ \operatorname{Stop}(A)<1.6+2.1<4$. This is a contradiction.

\subsection{Definition of the map $R$.}

5.5.1. Coordinates in a wall. The point $\xi \in \partial_{\infty} T$ defines an orientation on every edge $e \subset T$ by representing $e=v^{\prime} v$ with $B_{\xi}(v)=B_{\xi}\left(v^{\prime}\right)-1$. Depending on types of $v^{\prime}, v$ we classify the corresponding wall $W=W\left(v^{\prime}, v\right)$ as an $h h_{-}^{-}, s h_{-}^{-}, s s^{-}$, or $h s$-wall respectively. For instance, if $v^{\prime} \in S, v \in H$ then $W$ is an $s h$-wall etc. Recall that for every $s s$-wall the $\pi / 2$-condition is satisfied.

Given an $h h$ - or $s h$-wall $W=W\left(v^{\prime}, v\right)$ there is a unique point $w_{0} \in W$ satisfying the condition $\operatorname{dist}\left(w_{0}, W_{v}\right)=\operatorname{dist}\left(W, W_{v}\right)$ where we recall $W_{v} \neq W$ is the distinguished wall of the $H$-block $Y_{v}$. We fix mutually orthogonal $r$-line and $h$-line in $W$ passing through $w_{0}$ arbitrarily in the $h h$-case and letting the $r$-line be parallel to the $\mathbb{R}$-factor of $Y_{v^{\prime}}=F_{v^{\prime}} \times \mathbb{R}$ in the $s h$-case.

Let $W=W\left(v^{\prime}, v\right)$ be an $s s$-wall. We define its $h$-line $l_{h}(W) \subset W$ to be parallel to the $\mathbb{R}$-factor of the $S$-block $Y_{v}=F_{v} \times \mathbb{R}$ and singled out by the condition that its points minimize the distance to $W_{v}$ in $W$. The wall $W_{v}$ is either an $s h$-or $s s$-wall which has already defined $h$-line. In the block $Y_{v}$, this line defines an $\mathrm{H}^{2}$-section $F_{v} \times x_{v}$ of $Y_{v}=F_{v} \times \mathbb{R}$ by $l_{h}\left(W_{v}\right)=W_{v} \cap\left(F_{v} \times x_{v}\right)$. Now the $r$-line of $W$ is defined as $l_{r}(W)=W \cap\left(F_{v} \times x_{v}\right)$. We put $w_{0}=l_{r}(W) \cap l_{h}(W) \in W$.

We have defined an (non-oriented) coordinate system $\left(w_{0}, l_{r}, l_{h}\right)$ for each type of walls except the $h s$-type. However, for this type we do not need that.

5.5.2. Defining $R$. We fix a constant $D \geq 6$. Given $A \in \mathcal{C}_{0}$, we define $R(A) \in Y$ as follows. If $\operatorname{diam} \circ \operatorname{Stop}(A)<D$ then we pick $R(A) \in \operatorname{Stop}(A)$ arbitrarily. Now, we assume that diam $\circ \operatorname{Stop}(A) \geq D$.

First, consider the case when $\operatorname{Stop}(A)$ sits in an $S$-block $Y_{v}$. Then we have fixed the coordinate system $\left(w_{0}, l_{r}, l_{h}\right)$ in the wall $W_{v}$ because this wall is not of the $h s$-type. We let $R(A) \in \operatorname{Stop}(A)$ be a closest point to the $\mathrm{H}^{2}$-section $F_{v} \times x_{v} \subset Y_{v}=F_{v} \times \mathbb{R}$ through the coordinate line $l_{h}$.

By Proposition 5.13, we have $\operatorname{diam}(\operatorname{Stop}(A))_{\mathrm{H}^{2}} \leq 0.1$. Hence, $R(A)$ is defined up to 0.1 -errors in the $\mathrm{H}^{2}$-direction.

Assume finally that $\operatorname{Stop}(A)$ sits in an $H$-block $Y_{v}$. Then by Proposition 5.11, there is a wall $W \subset \partial Y_{v}$ different from $W_{v}$ which contains $\operatorname{Stop}(A)$. Thus $W$ is not an $h s$-wall, and the coordinate system $\left(w_{0}, l_{r}, l_{h}\right)$ is fixed in $W$. Moreover, recall that $\operatorname{Stop}(A)$ is the convex hull of the (proper) arc $\breve{A}:=\operatorname{Stop}(A) \cap S(\rho)$, where $S(\rho)=\partial W(\rho), W(\rho)=\left\{x \in W: \operatorname{dist}\left(x, W_{v}\right) \leq \rho\right\}, \rho \geq \rho_{0} ; S(\rho)$ is equidistant to $S\left(\rho_{0}\right)$ and $w_{0} \in W\left(\rho_{0}\right) \subset B_{r_{0}}^{W}\left(w_{0}\right)$ for some $r_{0}<2$.

Now, we proceed as follows. Consider a largest subarc $\breve{A}_{0} \subset \breve{A}$ with the same midpoint, which has no common interior point with the coordinate line $l_{r}$, and put $R(A) \in \breve{A}_{0}$ be the farthest point from $l_{r}$.

5.6. Proof of Theorem D: easy cases. We show that $R: \mathcal{C}_{0} \rightarrow Y$ is a local quasi-Lipschitz retraction, i.e., there exist $\varepsilon, \bar{D}>0$ such that $\operatorname{Hd}\left(A, A^{\prime}\right) \leq \varepsilon$ implies $\operatorname{dist}\left(R(A), R\left(A^{\prime}\right)\right) \leq \bar{D}$ for $A, A^{\prime} \in \mathcal{C}_{0}$ and $\operatorname{dist}(y, R(y)) \leq \bar{D}$ for $y \in Y$. Then $R_{1}=$ 
$R \circ R_{0}: \mathcal{C} \rightarrow Y$ is a local quasi-Lipschitz retraction by Lemma 5.9: take $\varepsilon^{\prime}<\varepsilon / 4, \varepsilon^{\prime \prime}=$ $\left(\varepsilon-4 \varepsilon^{\prime}\right) / L\left(\varepsilon^{\prime}\right)$. For $A, A^{\prime} \in \mathcal{C}$ with $\operatorname{Hd}\left(A, A^{\prime}\right) \leq \varepsilon^{\prime \prime}$ we have $\operatorname{Hd}\left(R_{0}(A), R_{0}\left(A^{\prime}\right)\right) \leq \varepsilon$, hence, $\operatorname{dist}\left(R_{1}(A), R_{1}\left(A^{\prime}\right)\right) \leq \bar{D}$. In addition $\operatorname{dist}\left(y, R_{1}(y)\right)=\operatorname{dist}(y, R(y)) \leq \bar{D}$ for each $y \in Y$.

The space $\mathcal{C}$ is geodesic, and for the geodesic spaces local quasi-Lipschitz implies quasi-Lipschitz, i.e., dist $\left(R_{1}(A), R_{1}\left(A^{\prime}\right)\right) \leq \frac{\bar{D}}{\varepsilon^{\prime \prime}} \operatorname{Hd}\left(A, A^{\prime}\right)$ for every $A, A^{\prime} \in \mathcal{C}$ with $\operatorname{Hd}\left(A, A^{\prime}\right) \geq \varepsilon^{\prime \prime}$.

Given $A, A^{\prime} \in \mathcal{C}_{0}$, we use notation $\widehat{A}:=\operatorname{Stop}(A)$ and $H:=\operatorname{Hd}\left(A, A^{\prime}\right)$. Furthermore, we assume that $H \leq \varepsilon<1 / 6$. By Corollary 5.6. we also can assume that

$$
\max \left\{\operatorname{diam} \widehat{A}, \operatorname{diam} \widehat{A}^{\prime}\right\} \geq D
$$

where $D \geq 6$ the constant from the definition of $R$.

5.6.1. Nonexceptional case of Proposition 5.5. By this Proposition $\widehat{A}, \widehat{A}^{\prime}$ are in one and the same block $Y_{v}$ and $\operatorname{Hd}\left(\widehat{A}, \widehat{A}^{\prime}\right) \leq 3 H \leq 3 \varepsilon<1 / 2$. Hence

$$
\left|\operatorname{diam} \widehat{A}-\operatorname{diam} \widehat{A}^{\prime}\right| \leq 2 \operatorname{Hd}\left(\widehat{A}, \widehat{A}^{\prime}\right)<1 \text {. }
$$

Now if $\operatorname{diam} \widehat{A}^{\prime}<D$ then $\operatorname{diam} \widehat{A} \leq D+1$, and we can apply Corollary 5.6 to obtain the result. Thus we assume that

$$
\min \left\{\operatorname{diam} \widehat{A}, \operatorname{dim} \widehat{A}^{\prime}\right\} \geq D .
$$

Subcase $(S): Y_{v}$ is an $S$-block, $Y_{v}=F_{v} \times \mathbb{R}$. In this case diam $\widehat{A}_{\mathrm{H}^{2}}, \operatorname{diam} \widehat{A}_{\mathrm{H}^{2}}^{\prime} \leq 0.1$ by Proposition 5.13. Since $D \geq 6$, this means that each of $\widehat{A}, \widehat{A}^{\prime}$ looks very much like a segment parallel to the $\mathbb{R}$-factor of $Y_{v}$, and we have actually 1-dimensional problem by projecting on the factors of $Y_{v}$. Thus

$$
\begin{aligned}
\operatorname{dist}\left(R(A), R\left(A^{\prime}\right)\right) & \leq \operatorname{dist}\left(R(A)_{\mathbb{R}}, R\left(A^{\prime}\right)_{\mathbb{R}}\right)+\operatorname{dist}\left(R(A)_{\mathrm{H}^{2}}, R\left(A^{\prime}\right)_{\mathrm{H}^{2}}\right) \\
& \leq 2 \operatorname{Hd}\left(\widehat{A}, \widehat{A}^{\prime}\right)+1 \leq 2
\end{aligned}
$$

according to the definition of $R$.

Subcase $(H): Y_{v}$ is an $H$-block. We postpone the discussion of this case to sect. 5.8.

5.6.2. Exceptional case of Proposition 5.5. In this case $A_{\mathrm{cr}}, A_{\mathrm{cr}}^{\prime}$ sit in one and the same block $Y_{v^{\prime}}$ and W.L.G. $\delta\left(A^{\prime}\right) \geq 5>\delta(A)$. Then $0<\delta\left(A^{\prime}\right)-\delta(A) \leq H$, $\widehat{A}^{\prime} \subset Y_{v^{\prime}}, \widehat{A} \subset Y_{v}$ with $B_{\xi}(v)=: k, B_{\xi}\left(v^{\prime}\right)=k+1$. Let $W=W\left(v^{\prime}, v\right)$ be the wall separating $Y_{v}$ and $Y_{v^{\prime}}$. Then $A_{k}, A_{k}^{\prime} \subset W$ and $\operatorname{Hd}\left(A_{k}, A_{k}^{\prime}\right) \leq H$ by Corollary 5.2. Furthermore $\operatorname{diam} \widehat{A}^{\prime} \geq \operatorname{diam} A_{k}^{\prime}$ and $\operatorname{diam} A_{k} \geq \operatorname{diam} \widehat{A}$. Hence if $\operatorname{diam} \widehat{A}^{\prime}<D$ then $\operatorname{diam} \widehat{A} \leq D+2 H<\bar{D}+1$ and we can apply Corollary 5.6.

Assume that $\operatorname{diam} \widehat{A}^{\prime} \geq D$. Then $\operatorname{diam} A^{\prime} \geq \operatorname{diam} \widehat{A}^{\prime}>1$ and $Y_{v^{\prime}}$ is an $S$-block since otherwise $\delta\left(A^{\prime}\right) \geq 10$ by Lemma 5.12. This contradicts $\delta\left(A^{\prime}\right) \leq \delta(A)+H<10$. Hence $Y_{v^{\prime}}=F_{v^{\prime}} \times \mathbb{R}$ and we have $\operatorname{diam} \widehat{A}_{\mathrm{H}^{2}}^{\prime} \leq 0.1$ by Proposition 5.13 and similarly $\operatorname{diam}\left(A_{k}\right)_{\mathrm{H}^{2}} \leq 0.1$. Furthermore

$$
\begin{aligned}
\operatorname{Hd}\left(\widehat{A}^{\prime}, A_{k}\right) & \leq \operatorname{Hd}\left(\widehat{A}^{\prime}, A_{k}^{\prime}\right)+\operatorname{Hd}\left(A_{k}^{\prime}, A_{k}\right) \\
& \leq \delta\left(A^{\prime}\right)-5+H \leq 2 H .
\end{aligned}
$$

Let $\left(w_{0}, l_{r}, l_{h}\right)$ be the coordinate system in the wall $W$. Then for a point $b \in$ $A_{k}$ closest to $l_{h}$ we have $\operatorname{dist}\left(R\left(A^{\prime}\right), b\right) \leq 2 \operatorname{Hd}\left(\widehat{A}^{\prime}, A_{k}\right)+1 \leq 1+4 \varepsilon$ exactly as in 
Subcase $(S)$. On the other hand, it follows from the definition of $l_{h}$ and the touching point $\tau_{k}$ (see sect. 5.1.3) that $b \in \zeta_{A}\left(\tau_{k}\right)$. Furthermore, we have $\operatorname{Hd}\left(\zeta_{A}\left(\tau_{k}\right), \widehat{A}\right) \leq$ $5-\delta(A) \leq \delta\left(A^{\prime}\right)-\delta(A) \leq H$ by the definition of $\widehat{A}=\operatorname{Stop}(A)$, in particular, $\operatorname{dist}(b, \widehat{A}) \leq H$.

Assume that $\operatorname{diam} \widehat{A}<D$. Then $\operatorname{dist}(b, R(A)) \leq H+D$ and we obtain

$$
\operatorname{dist}\left(R(A), R\left(A^{\prime}\right)\right) \leq \operatorname{dist}(R(A), b)+\operatorname{dist}\left(b, R\left(A^{\prime}\right)\right) \leq D+2 .
$$

The remaining case $\operatorname{diam} \widehat{A}^{\prime}, \operatorname{diam} \widehat{A} \geq D$ will be considered in sect. 5.8 .

5.7. Digression: a Lipschitz extension property for $S^{1}$. Let $S_{\rho}^{1} \subset \mathbb{R}^{2}$ be a circle of radius $\rho$ endowed with the induced intrinsic metric. We define $\mathcal{C}\left(S_{\rho}^{1}\right)$ to be the set of all proper $\operatorname{arcs} A \subset S_{\rho}^{1}$. This set can be identified with $S_{\rho}^{1} \times[0, \pi \rho)$ via $A \mapsto(x, r)$, where $x \in A$ is the midpoint and $r$ the half of the length of $A$. We assume that $\mathcal{C}\left(S_{\rho}^{1}\right)$ is endowed with the metric induced from $S_{\rho}^{1} \times[0, \pi \rho)$ by that identification.

Fix a line $l \subset \mathbb{R}^{2}$ through the center $w_{0}$ of $S_{\rho}^{1}$ and consider the subset $\Phi \subset \mathcal{C}\left(S_{\rho}^{1}\right)$ which consists of all $A$ having no common interior point with $l$. We define a retraction $f: \Phi \rightarrow S_{\rho}^{1}$ letting $f(A)$ be the point of $A \in \Phi$ of maximal distance to $l$. Next we extend $f$ to $\bar{f}: \mathcal{C}\left(S_{\rho}^{1}\right) \rightarrow S_{\rho}^{1}$ by taking the largest subarc $A_{0} \subset A$ with the same midpoint, $A_{0} \in \Phi$ and putting $\bar{f}(A)=f\left(A_{0}\right)$, cp. the last paragraph of sect. 5.5.2.

LEMMA 5.14. The retraction $f: \Phi \rightarrow S_{\rho}^{1}$ is $\sqrt{2}$-Lipschitz and its extension $\bar{f}: \mathcal{C}\left(S_{\rho}^{1}\right) \rightarrow S_{\rho}^{1}$ is 2-Lipschitz.

Proof. This is clear from Figure 5.1 where the left and the right vertical segments are identified, $\{a, b\}=l \cap S_{\rho}^{1}$ and $\Phi$ consists of two equilateral triangles (with interiors) drawn in bold.



FIG. 5.1. the map $\bar{f}$

5.8. Proof of Theorem D: difficult cases. We use the notations introduced in sect. 5.6. First consider the postponed

Subcase $(H): \widehat{A}, \widehat{A}^{\prime}$ are sitting in an $H$-block $Y_{v}, \operatorname{diam} \widehat{A}, \operatorname{diam} \widehat{A}^{\prime} \geq D$ and $\operatorname{Hd}\left(\widehat{A}, \widehat{A}^{\prime}\right) \leq 3 H \leq 3 \varepsilon$. Then by Proposition $5.11, \widehat{A}, \widehat{A}^{\prime}$ are in a wall $\bar{W} \subset \partial Y_{v}$ different from $W_{v}$ and moreover each of $\widehat{A}, \widehat{A}^{\prime}$ is the convex hull of an arc on $\partial W(\rho)$, $\partial W\left(\rho^{\prime}\right)$ respectively, where $W(\rho)=\left\{x \in W: \operatorname{dist}\left(x, W_{v}\right) \leq \rho\right\}$. Then, obviously, $\left|\rho-\rho^{\prime}\right| \leq \operatorname{Hd}\left(\widehat{A}, \widehat{A}^{\prime}\right) \leq 3 H$. 
Since $W$ is an $s h$ - or $h h$-wall, the coordinate system $\left(w_{0}, l_{r}, l_{h}\right)$ in $W$ is fixed. Recall that $\partial W(\rho)$ is equidistant to $\partial W\left(\rho_{0}\right)$ and $W\left(\rho_{0}\right) \subset B_{r_{0}}^{W}\left(w_{0}\right)$ for $r_{0}<2$, where $W\left(\rho_{0}\right)$ is the smallest $W(\rho)$ containing $\bar{K}$ (see sect. 5.4.1). We have

$$
\widehat{A} \subset W(\rho)=W\left(\rho_{0}+\rho-\rho_{0}\right) \subset B_{r_{0}+\rho-\rho_{0}}^{W}\left(w_{0}\right) .
$$

Thus diam $\widehat{A} \leq 2\left(r_{0}+\rho-\rho_{0}\right)$ and hence $\rho-\rho_{0}, \rho^{\prime}-\rho_{0} \geq D / 2-r_{0}>1$. We also note that $\breve{A}=\widehat{A} \cap \partial W(\rho)$ is a proper arc as it is easy to see from the definition of a stopping set.

To make the argument clear we simplify the situation assuming that $\bar{K} \subset W$ is a ball centered at $w_{0}$. This is the case when the 1-neighbourhood of the shortest segment between $W$ and $W_{v}$ intersects no other wall of $Y_{v}$.

In this case $\partial W(\rho)$ is a circle in $W$ of radius $\geq D / 2-r_{0}$ centered at $w_{0}$. Furthermore, $\breve{A}$ subtends the angle at most $\pi$ at $w_{0}$. Note that the estimates of Lemma 5.14 are independent of $\rho$ and the construction of $\bar{f}$ from this Lemma is equivariant w.r.t. the homotheties centered at $w_{0}$. Thus taking into account the estimate $\left|\rho-\rho^{\prime}\right| \leq 3 H$ and applying Lemma 5.14 we easily obtain dist $\left(R(A), R\left(A^{\prime}\right)\right) \leq L \cdot H$ for some $L>0$. Besides Lemma 5.14 and the mentioned estimate, the main contribution in $L$ is due to the transition from the Hausdorff metric on subsets in $W$ to the metric used in this Lemma. However, this is bilipschitz for $\varepsilon$ sufficiently small and the estimate $L<100$ would be too pessimistic.

In general case when $\bar{K}$ is not supposed to be a ball, the constant $L$ is spoiled but only by a bounded (multiplicative) amount. The reason is that the main danger for $L$ comes from the possibility of $\breve{A}$ to subtend an angle at $w_{0}$ close to $2 \pi$, i.e., when $\widehat{A}$ almost coincides with $W(\rho)$. However, this angle though might be bigger than $\pi$ is then arbitrarily close to $\pi$ for all sufficiently large $D$ because $\partial W(\rho)$ is contained in the annulus $\left\{x \in W: \rho-\rho_{0} \leq \operatorname{dist}\left(x, w_{0}\right) \leq r_{0}+\rho-\rho_{0}\right\}$ with $\rho-\rho_{0} \geq D / 2-r_{0}$ and recall $W(\rho)$ is convex.

It remains to consider the exceptional case of Proposition 5.5 when both $\operatorname{diam} \widehat{A}$, $\operatorname{diam} \widehat{A}^{\prime} \geq D$. As in sect. 5.6.2 we assume that $\delta\left(A^{\prime}\right) \geq 5>\delta(A)$ and use all agreements and notations of that section. Then $Y_{v}$ is an $H$-block by Proposition 5.13 because $5>\delta(A) \geq \delta\left(A^{\prime}\right)-H>4$. Consequently, $W=W\left(v^{\prime}, v\right)$ is an sh-wall and $\widehat{A} \subset W$ by Proposition 5.11. Recall also that dist $\left(R\left(A^{\prime}\right), b\right) \leq 1+4 \varepsilon$ for $b \in A_{k}$ closest to the coordinate line $l_{h}$ of the coordinate system $\left(w_{0}, l_{r}, l_{h}\right)$ in $W$ and $\operatorname{diam}\left(A_{k}\right)_{\mathrm{H}^{2}} \leq$ 0.1 , i.e., $A_{k} \subset W$ is a segment of length $\geq D$ parallel to $l_{r}$ (up to 0.1 -errors which we ignore in the sequel). Furthermore, $b \in \zeta_{A}\left(\tau_{k}\right)$ and $\operatorname{Hd}\left(\zeta_{A}\left(\tau_{k}\right), \widehat{A}\right) \leq H$.

Note that $b$ is the remotest point from $l_{r}$ in $\zeta_{A}\left(\tau_{k}\right)$ because $\zeta_{A}\left(\tau_{k}\right)$ touches $A_{k}$ at $b$ and $A_{k}$ is parallel to $l_{r}$.

For the same reason as above we assume that $\bar{K} \subset W$ is a round ball centered at $w_{0}$. Then $\zeta_{A}\left(\tau_{k}\right)$ and consequently $\breve{A}=\widehat{A} \cap W(\rho)$ have no common interior point with $l_{r}$. By the definition of $R$ we have $R(A) \in A$ is a point closest to $b$ and thus $\operatorname{dist}(R(A), b) \leq \operatorname{Hd}\left(\widehat{A}, \zeta_{A}\left(\tau_{k}\right)\right) \leq H$. Hence $\operatorname{dist}\left(R(A), R\left(A^{\prime}\right)\right) \leq 1+4 \varepsilon+H<2$.

The general case that $\bar{K}$ is not a ball may cause that $\breve{A}$ has a common interior point with $l_{r}$. However, it may effect the estimate for $\operatorname{dist}(R(A), b)$ only by an additive amount bounded by $r_{0}$. This again follows from the fact that $\partial W(\rho)$ sits in the annulus centered at $w_{0}$ which has a large diameter $\geq D / 2-r_{0}$ and a bounded width $\leq r_{0}$. This completes the proof of Theorem D. 


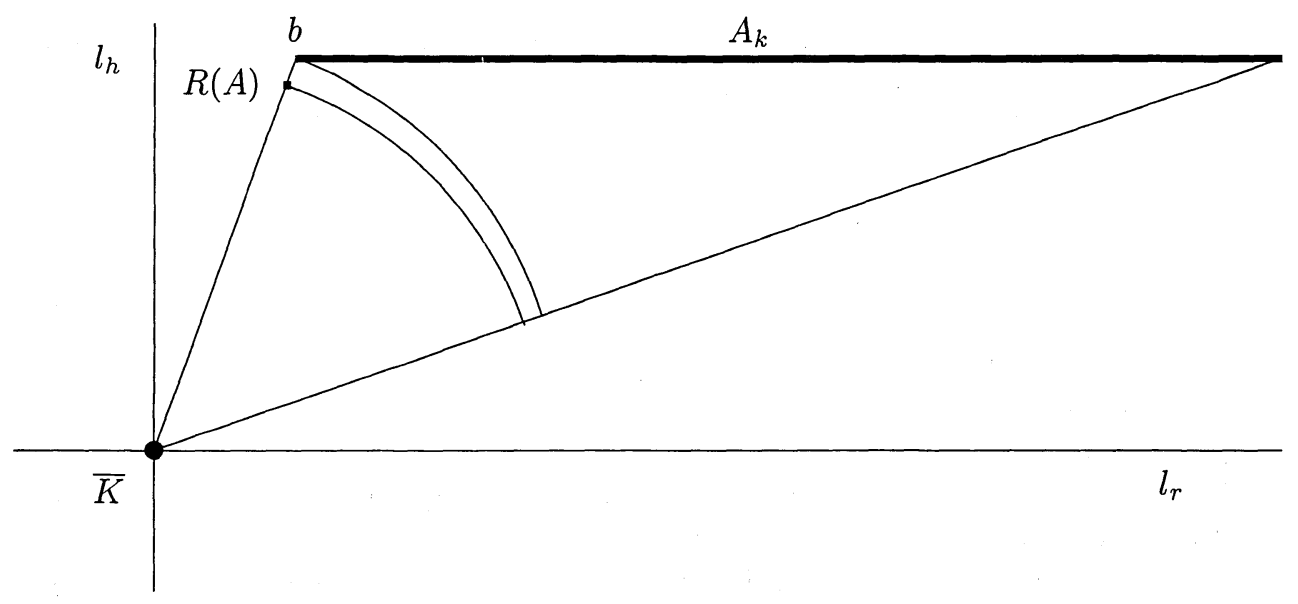

FIG. 5.2. farthest points from $l_{r}$

\section{REFERENCES}

[BrH] M. Bridson, A. Haefliger, Metric spaces of non-positive curvature, Springer, Berlin Heidelberg, 1999.

[B] S. BUYALO, Euclidian planes in three-dimensional manifolds of nonpositive curvature, Math. Notes, 43 (1988), pp. 60-66.

[BK] S. BUYALO, V. KoBEL'SKII, Geometrization of graph-manifolds. II. Isometric geometrization, St. Petersburg Math. J., 7 (1996), pp. 387-404.

[Eetal] D. Epstein, J. Cannon, D. Holt, S. Levy, M. Paterson, W. Thurston, Word-Processing in groups, Barlett and Jones, Boston 1992.

[Eb] P. EberLein, Geodesic flow in certain manifolds without conjugate points, Trans. AMS, 167 (1972), pp. 151-170.

[KLe] M. KAPOVICH AND B. LEEB, 3-manifold groups and nonpositive curvature, Geom. funct. anal., 8 (1998), pp. 841-852.

[L] U. LANG, Extendability of large scale lipschitz maps, Trans. AMS, 351(1999), pp. 3975-3988.

[LPS] U. LANG, B. PAVlović, V. Schroeder, Extensions of Lipschitz maps into Hadamard spaces, Geom. funct. anal., 10 (2000), pp. 1527-1553.

[Le] B. LeEB, 3-manifolds with(out) metrics of nonpositive curvature, Invent. Math., 122 (1995), pp. $277-289$.

[M] E.J. MCShane, Extension of range of functions, Bull. AMS, 40 (1934), pp. 837-842.

[Sch] R. SCHNEIDER, Convex Bodies: The Brunn - Minkowski Theory, Cambridge University Press, 1993.

[S] V. Schroeder, Codimension one tori in manifolds of nonpositive curvature, Geometriae Dedicata, 33 (1990), pp. 251-263. 